\title{
Annual modulation in the scattering of J1819+3845: Peculiar plasma velocity and anisotropy
}

\author{
J. Dennett-Thorpe ${ }^{1,2}$ and A. G. de Bruyn ${ }^{1,2}$ \\ 1 Netherlands Foundation for Research in Astronomy, Postbus 2, 7990 AA, Dwingeloo, The Netherlands \\ 2 Kapteyn Institute, Postbus 800, 9700 AV Groningen, The Netherlands \\ Received 26 June 2002 / Accepted 28 February 2003
}

\begin{abstract}
We present two years of monitoring observations of the extremely variable quasar J1819+3845. We observe large yearly changes in the timescale of the variations (from $\sim 1$ hour to $\sim 10$ hours at $5 \mathrm{GHz}$ ). This annual effect can only be explained if the variations are caused by a propagation effect, and thus affected by the Earth's relative speed through the projected intensity pattern. To account for this effect, the scattering plasma must have a transverse velocity with respect to the local standard of rest. The velocity calculated from these observations is in good agreement with that obtained from a two telescope delay experiment (Dennett-Thorpe \& de Bruyn 2001). We also show that either the source itself is elongated, or that the scattering plasma is anisotropic, with an axial ratio of $>6: 1$. As the source is extended on scales relevant to the scattering phenomenon, it seems plausible that the anisotropy is due to the source itself, but this remains to be investigated. From the scintillation characteristics we find that the scattering material is a very strong, thin scatterer within $\sim$ ten parsecs. We determine a source size at $5 \mathrm{GHz}$ of 100 to 900 microarcsecs, and associated brightness temperatures of $10^{10}$ to $10^{12} \mathrm{~K}$.
\end{abstract}

Key words. scattering - ISM: structure - quasars: individual: J1819+3845 - radio continuum: galaxies - techniques: high angular resolution

\section{Introduction}

Variability in radio sources can be due to either intrinsic variations in the source itself, or due to a propagation effect known as scintillation. Disentangling these two effects is critical to our understanding of the central regions of quasars and radio galaxies, as in both cases the variability gives dimensions of the emitting region, but the interpretation (and the implied size) are quite different.

Intrinsically variable extragalactic radio sources include blazars, which exhibit changes in flux density of up to an order of magnitude or more occurring over decades (Aller et al. 1985), and smaller outbursts on shorter timescales (e.g. Pohl et al. 1996; Stevens et al. 1995). Intraday variability is a phenomenon at $\mathrm{GHz}$ frequencies with typically a few percent variations on day timescales (e.g. Heeschen et al. 1987; Quirrenbach et al. 2000). An intrinsic explanation requires the sources to have brightness temperatures far in excess of $10^{12} \mathrm{~K}$ - up to $10^{17}-10^{19} \mathrm{~K}$.

Scintillation occurs due to changes in electron density in the intervening material. Interplanetary scintillation is caused by the solar wind. The variations of the flux density of quasars close to the Sun was used to deduce that some quasars had components of arcsecond size (Hewish et al. 1964; Cohen et al. 1967; Readhead \& Hewish 1976) - a claim since confirmed by radio interferometry. Interstellar scintillation is caused by

Send offprint requests to: J. Dennett-Thorpe, e-mail: jdennett@diversity-radio.net the material throughout the Galaxy, and is responsible both for many effects seen in pulsars (Rickett 1990, and references therein) and low-frequency variability (LFV) of some extragalactic radio sources (Hunstead 1972). The compact structure in LFVs is expected to be of the order of a few milliarcsec, and is indeed seen in VLBI observations (e.g. Spangler et al. 1993). The scintillation regimes generally appropriate for extragalactic sources are the (broad-band) weak and refractive regimes. (Pulsar observations are generally concerned with narrow-band diffractive phenomena. See Narayan 1992 for a review of scintillation).

Although refractive ISS is now commonly believed to be a major contributor to the variability at low frequencies (Spangler et al. 1989, 1993; Bondi et al. 1996), as first suggested by Rickett et al. (1984), its relevance to observed variations at $\mathrm{GHz}$ frequencies has been the subject of some debate (Wagner \& Witzel 1995), in particular because it cannot account for the reported correlation between optical and radio variability, which have been observed in a few sources (Wagner et al. 1996; Peng et al. 2000). The origin of the IDV in B $0917+624$, one of the most variable classical IDV sources, with $10-15 \%$ variations, has been discussed by a variety of authors with different views (e.g. Quirrenbach et al. 1989; Qian et al. 1991; Simonetti 1991; Shapirovskaya 1991; Rickett et al. 1995; Kraus et al. 1999). An intrinsic explanation for the variability in this source gives an apparent brightness temperature of $10^{18} \mathrm{~K}$, which can be brought under the Compton limit by a Doppler factor of $\sim 17$ (Quirrenbach et al. 1989). 
PKS 0405-385 was the first extragalactic radio source to show variations at $\mathrm{GHz}$ frequencies that were fairly unambiguously interpreted as due to ISS (Kedziora-Chudczer et al. 1997). Using the simplest assumptions about the location and the velocity of the scatterer, Kedziora-Chudzner et al. calculated the brightness temperature of PKS 0405-385 to be $5 \times$ $10^{14} \mathrm{~K}$, or 1000 times the inverse Compton limit. However, this figure depends critically on the assumptions about the scatterer.

In this paper we investigate the properties of the flux density variations of the quasar $\mathrm{J} 1819+3845$. The variations in this source are the most extreme known in the radio sky. We previously interpreted the variability as due to interstellar scintillation (ISS), based on the extreme nature of the variations and their frequency dependence (Dennett-Thorpe \& de Bruyn 2000, hereafter Paper I). We have since proved them to be due to ISS by detecting a difference in the arrival times (of up to $100 \mathrm{~s}$ ) of the flux density variations at two widely spaced telescopes (Dennett-Thorpe \& de Bruyn 2001, hereafter Paper II).

In Paper I we calculated the location of the scatterer using simple formulae and the observed timescale, depth of the intensity variations and the frequency of maximum variations. We assumed that the scatterer was at rest w.r.t. the LSR, and thereby calculated in a straightforward manner that the brightness temperature of the scintillating part of J1819+3845 was $\geq 5 \times 10^{12} \mathrm{~K}$. This is an order of magnitude higher than the Compton limit - a fact which could be explained by Doppler boosting, but this expects rapid energy loss at higher radio frequencies. Furthermore all known sources with Doppler factors of 10 or more are themselves intrinsically variable.

We conducted a monitoring campaign on $\mathrm{J} 1819+3845$ to determine the longevity and stability of the source and the velocity of the scatterer (an unknown in the $T_{B}$ calculation). Since the programme started we have measured the velocity of the scattering plasma by another means (Paper II). With the monitoring campaign we also wished to try to "resolve" the source by observing a change in scintillation characteristics throughout the year, as we sampled the source along different axes. Here we report on the monitoring campaign at $5 \mathrm{GHz}$ and will use the conclusions in later papers which will discuss the (multi-frequency) structure of the source.

In Sect. 2 we describe the observations (and include an optical spectrum). We give an overview of the results in Sect. 3 before undertaking an analysis of the light-curves (Sect. 4). Sections 5 and 6 give the interpretation of the results by fitting models for plasma velocity and anisotropy to the data. In Sects. 7 and 8 give give some further consideration to the scattering medium and source stability. In Sect. 9 we derive the parameters of the scattering medium, and the size of the source, taking into consideration effects of source resolution. We end with a summary of the results and concluding remarks.

\section{Observations}

\subsection{Radio}

The observations were conducted with the Westerbork Synthesis Radio Telescope (WSRT) in the period May 1999 to July 2001. The details of the observations are given in Table 1 . The telescope generally observed in full array mode, with all antennae working at the same frequency, but on a number of occasions two frequencies were observed separately in split-array mode. From December 2000 onwards most observations were taken in split-array configurations with 5 telescopes at $2.3 \mathrm{GHz}, 4$ telescopes at $4.9 \mathrm{GHz}$ and 5 telescopes at $8.5 \mathrm{GHz}$. The number of telescopes used at $4.9 \mathrm{GHz}$ is indicated in Col. 4 of Table 1. (Data at the other frequencies will be presented and analysed as part of a forthcoming paper.) On-line integration times were set to $10 \mathrm{~s}$. The total recorded bandwidth was $80 \mathrm{MHz}$, in 8 contiguous $10 \mathrm{MHz}$ bands. In the split-array observations software constraints limited the number of independent bands to $6 \times 10 \mathrm{MHz}$.

Most observations consisted of long, uninterrupted tracks, of up to $12 \mathrm{~h}$ duration. Each long track was preceded and followed by short observations of polarized and unpolarized calibrators (generally 3C 286/CTD 93 before and 3C 48/3C 147 afterwards). Absolute flux density calibration was performed assuming a flux density of $7.42 \mathrm{Jy}$ for 3C 286 and $1.70 \mathrm{Jy}$ for CTD 93 at a frequency of $4.874 \mathrm{GHz}$. The polarisation characteristics will be dealt with in a later paper.

The reduction package NEWSTAR (Noordam et al. 1994) was used to calibrate and examine the data. Bad bands, telescopes and baselines were excised where necessary. The amplitude and phase calibration solutions determined for the calibrator were applied to $\mathrm{J} 1819+3845$. The amplitude stability of the WSRT is generally very good. The errors generally contain three components which are due to pointing $(<1 \%$ at $4.9 \mathrm{GHz}$ ), system temperature and atmospheric opacity variations. The main observed variations in $T_{\text {sys }}$ are due to variable spillover losses and atmospheric emission, both of which vary only slowly. The WSRT continuously measures $T_{\text {sys }}$ from stable noise sources, allowing these slow variations to be accurately removed, resulting in an overall array amplitude stability of better than $1 \%$ over periods in excess of 12 hours.

The largest remaining uncertainty is due to the atmospheric opacity variations associated with (heavy) rainfall. The emission effects on the $T_{\text {sys }}$ are taken care of but the associated opacity effects are not. The uncertainty in the flux density scale due to opacity effects is less than $1 \%$. Errors associated with heavy rainfall, occurring less than $1 \%$ of the time, can, however, lead to an underestimate of the flux density of a few percent.

Phase self-calibration was applied to the observations of $\mathrm{J} 1819+3845$, with a $5 \mathrm{~min}$ averaging interval. The source model of $\mathrm{J} 1819+3845$ at $4.9 \mathrm{GHz}$ was a single point source at the phase centre, as there are no sources detected above $1 \mathrm{mJy}$ within the primary beam. Previous observations with the VLA had already confirmed that there is no structure in the source on scales in between 0.3 and $3^{\prime \prime}$, the resolution of the WSRT at $4.9 \mathrm{GHz}$. The source flux density was then calculated from the real part of the complex visibility, averaged over the desired sampling interval (30 or 120 s). This requires that the phase is stable over the period of the self-calibration solution, a condition seen to be held from inspection of the slowly changing phase solutions. This method has the advantage that it does not require a noise bias correction which is necessary if scalar averaging would be used. Rare occasions of 
Table 1. Observational parameters.

\begin{tabular}{|c|c|c|c|c|c|}
\hline Date & $\begin{array}{l}\text { day } \\
\text { number }\end{array}$ & MJD & $\begin{array}{l}\text { duration } \\
\text { (hrs) }\end{array}$ & $\begin{array}{l}\text { number } \\
\text { tels }\end{array}$ & notes \\
\hline 1999-05-13 & 133 & 51311 & 12 & 7 & Paper I \\
\hline 1999-05-14 & 134 & 51312 & 12 & 7 & Paper I \\
\hline 1999-06-21 & 172 & 51350 & 2.5 & 14 & \\
\hline 1999-07-02 & 183 & 51361 & 2 & 14 & \\
\hline 1999-07-05 & 186 & 51364 & 5.5 & 14 & \\
\hline 1999-07-17 & 198 & 51376 & 6 & 14 & \\
\hline 1999-07-30 & 211 & 51389 & 6 & 14 & \\
\hline 1999-08-13 & 225 & 51403 & 12 & 7 & \\
\hline 1999-08-27 & 239 & 51417 & 6 & 14 & \\
\hline 1999-09-07 & 250 & 51428 & 12 & 14 & \\
\hline 1999-09-21 & 264 & 51442 & 5 & 14 & \\
\hline 1999-09-25 & 268 & 51446 & 5 & 14 & \\
\hline 16-10-1999 & 289 & 51467 & 4 & 14 & \\
\hline 1999-10-29 & 302 & 51480 & 7 & 14 & \\
\hline 1999-11-28 & 332 & 51510 & 6.5 & 14 & \\
\hline 1999-12-11 & 345 & 51523 & 8.5 & 7 & \\
\hline 1999-12-30 & 364 & 51542 & 12 & 7 & \\
\hline $2000-01-21$ & 21 & 51564 & 12 & 7 & \\
\hline 2000-02-05 & 36 & 51579 & 12 & 14 & \\
\hline 2000-02-29 & 60 & 51603 & 2 & 14 & \\
\hline 2000-03-09 & 69 & 51612 & 2 & 14 & \\
\hline 2000-03-18 & 78 & 51621 & 12 & 14 & \\
\hline 2000-04-14 & 106 & 51648 & 9 & 14 & \\
\hline 2000-05-14 & 135 & 51678 & 6 & 14 & \\
\hline 2000-05-28 & 149 & 51692 & 8.5 & 14 & \\
\hline 2000-06-23 & 175 & 51718 & 12 & 14 & \\
\hline 2000-07-26 & 208 & 51751 & 12 & 14 & \\
\hline 2000-08-09 & 222 & 51765 & 12 & 14 & \\
\hline 2000-08-27 & 240 & 51783 & 12 & 14 & \\
\hline 2000-09-08 & 252 & 51795 & 12 & 14 & \\
\hline 2000-10-13 & 287 & 51830 & 10 & 14 & \\
\hline 2000-11-25 & 330 & 51873 & 12 & 7 & \\
\hline $2000-12-10$ & 345 & 51888 & 12 & 4 & \\
\hline 2000-12-26 & 361 & 51904 & 12 & 4 & \\
\hline 2001-01-07 & 007 & 51916 & 6 & 14 & Paper II \\
\hline 2001-01-12 & 012 & 51921 & 6 & 14 & Paper II \\
\hline 2001-03-17 & 076 & 51985 & 12 & 4 & \\
\hline 2001-03-31 & 090 & 51999 & 12 & 4 & \\
\hline $2001-05-20$ & 140 & 52049 & 12 & 4 & \\
\hline 2001-06-07 & 158 & 52067 & 9 & 4 & \\
\hline $2001-06-10$ & 161 & 52070 & 12 & 4 & \\
\hline 2001-07-02 & 183 & 52092 & 12 & 4 & \\
\hline 2001-07-24 & 205 & 52114 & 12 & 4 & \\
\hline month & \multicolumn{3}{|c|}{ day numbers } & duration & telescopes \\
\hline 1999-09 & \multicolumn{3}{|c|}{$\begin{array}{l}244,245,246,247,248 \\
252,254,256,261,262\end{array}$} & $10 \mathrm{~min}$ & 14 \\
\hline 2000-09 & \multicolumn{3}{|c|}{$244,245,246,247,248,249$} & $3 \mathrm{~min}$ & 14 \\
\hline $2000-10$ & \multicolumn{3}{|c|}{$300,301,302,303,306$} & $3 \mathrm{~min}$ & 14 \\
\hline
\end{tabular}

rapid atmospherically induced phase variations, were detected by comparison with the scalar averaged data: on $27 / 08 / 1999$, 08/09/2000 and 13/10/2000, when the phase fluctuations result in a minor (a couple of percent) time-dependent variation in the calculated flux density.

Typically between 70 and 80 baselines contribute to the final datum. With an rms noise of $20 \mathrm{mJy}$ per baseline, for $120 \mathrm{~s}$ averaging times in one $10 \mathrm{MHz}$ band, we then achieved a typical noise in the source flux density of about $0.8 \mathrm{mJy}$ for eight $10 \mathrm{MHz}$ bands and $120 \mathrm{~s}$ integration. This $0.8 \mathrm{mJy}$ is the thermal noise error. For the 4 telescope ( 6 baseline), $60 \mathrm{MHz}$ observations from December 2000 onwards the error is about 4 times larger, i.e. $3 \mathrm{mJy}$. The final flux density error is the quadrature sum of a thermal error and the slowly varying gain error discussed above which we estimated at typically $1 \%$. With a median flux density of the source of $200 \mathrm{mJy}$ we can see that the overall error is never more than about $1.5 \%$. The smooth light curves, in periods of slow scintillations, show that our estimations of the errors are realistic.

For the 10 min observations conducted in September 1999, and in two periods in September 2000 and November 2000, the errors in the absolute flux density scale outweigh the errors from the fit.

\subsection{Optical}

A spectrum was obtained at the WHT on 17 July 2000 in three $1800 \mathrm{~s}$ exposures. The ISIS spectrograph was used to obtain a spectrum from $3000 \AA$ to $8000 \AA$, with a resolution of 0.86 and $2.9 \AA /$ pixel and central wavelengths of $4495 \AA$ and $6708 \AA$ on the blue and red arms respectively. Standard reduction procedures were followed using the IRAF longslit software package. The combined spectrum, with the blue arm boxcar smoothed over 9 pixels, is shown in Fig. 1. The fitted line properties are shown in Table 2.

We calculate the redshift to be $0.533 \pm 0.001$. The spectrum is fairly typical for quasars, both in the equivalent widths of the lines and in the continuum emission (see e.g. Miller et al. 1992; Baker 1997; Brotherton et al. 2001). We note the relatively small Balmer decrement $(\mathrm{H} \beta / \mathrm{H} \gamma)$, possibly indicating little line of sight obscuration by dust. The [OII] luminosity $\left(10^{34} \mathrm{~W}\right)$ is comparable to moderately powerful FRII radio sources (radio luminosities at $151 \mathrm{MHz}$ of $10^{25}-10^{27} \mathrm{~W} / \mathrm{Hz} / \mathrm{str}$ ) (Willott et al. 1999). We have observed the optical spectrum of this source twice. On both ocassions the optical magnitude was similar, and the lines strong and broad. From these observations it does not seem that $\mathrm{J} 1819+3845$ is a typical OVV; rather a typical quasar.

\section{Overview of radio observations and comments on individual epochs}

In Fig. 2 we present the light-curves over more than two years of observations. In these curves the averaging time is $120 \mathrm{~s}$. For the analysis we used $30 \mathrm{~s}$ integrations, an example of which is plotted in Fig. 3. The interrupted light curves in September 1999 are due to rapid switching between two frequencies about 100-200 MHz apart. 


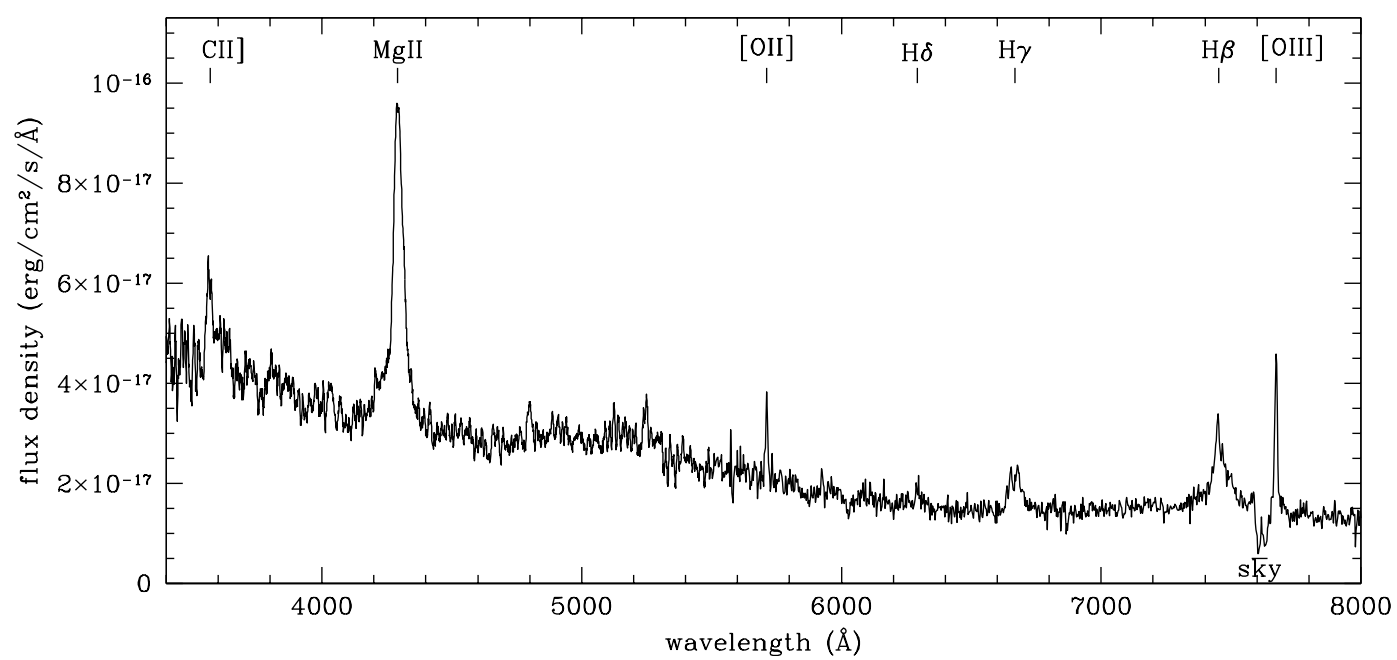

Fig. 1. WHT spectrum of J1819+3845, with identified lines indicated.

Table 2. Fitted line properties of optical spectrum.

\begin{tabular}{lllrrl}
\hline \hline lines & $\begin{array}{l}\lambda \\
(\AA)\end{array}$ & $\begin{array}{l}E W_{\text {rest }}^{\text {(int) }} \\
(\AA)\end{array}$ & $\begin{array}{r}E W_{\text {rest }}^{\text {(fit) }} \\
(\AA)\end{array}$ & $\begin{array}{l}f \\
\left(\mathrm{erg} / \mathrm{s} / \mathrm{cm}^{2}\right)\end{array}$ \\
\hline$[\mathrm{CII}]$ & 3565.5 & 1.0 & 8 & 7 & $3.7 \mathrm{e}-16$ \\
$\mathrm{MgII}$ & 4292.8 & 0.3 & 73 & 69 & $2.4 \mathrm{e}-15$ \\
{$[\mathrm{OII}]$} & 5712.1 & 0.3 & 5 & 5 & $1.1 \mathrm{e}-16$ \\
$\mathrm{H} \delta$ & 6293.2 & 2.0 & 5 & 3 & $0.8 \mathrm{e}-16$ \\
$\mathrm{H} \gamma$ & 6668.1 & 2.0 & 22 & 21 & $3.2 \mathrm{e}-16$ \\
$\mathrm{H} \beta$ & 7452.2 & 2.0 & 51 & 45 & $7.7 \mathrm{e}-16$ \\
{$[\mathrm{OIII}]$} & 7673.5 & 0.3 & 20 & 19 & $2.8 \mathrm{e}-16$ \\
\hline
\end{tabular}

The rapid variations of May 1999 have become slower by June and July of that year, although they still are fast enough to show complete modulations in a few hours. By the end of August the modulations have become so slow that the source remains at an almost constant flux density over 6 hours. The fact that the source is still varying, but with a very long timescale can be seen in Fig. 5, which shows the variations over August and September, including the short ( $\sim 10$ mins) observations taken over the ten days following the quasi-stationary observations on August 27 (day 239). The source was observed close to its minimum flux density $(60 \mathrm{mJy})$ on the next observation (September 1, day 244), and on the following day at a flux density typical of the peak excursions $(220 \mathrm{mJy})$.

The modulations remain slow until December, when they quickly speed up again. By the end of 1999 there are rapid intensity variations with peak to peak timescales of around one and a half hours. The variations remain fast, up to and including the observations of 15 May 2000, one year after the first twelve hour observations. It can be seen that the character and timescale of the observations is extremely similar to the observations of one year previously.

The observations during May 2000 to July 2001 broadly replicate the observations of the previous year: showing a slowing down of the variations between June and December, before speeding up again. Note also the very rapid speeding up of the scintillations in December of both years: a factor of 2 within an interval of just 3 weeks.

We have analyzed variations across the $80 \mathrm{MHz}$ bands and found them highly correlated. The correlation coefficients of $>0.99$ are consistent with completely correlated signals with thermal noise. At $5 \mathrm{GHz}$ the scintillation is clearly broad-band (Fig. 4).

\section{Analysis of light curves}

\subsection{Modulation index}

The modulation index is the normalized rms intensity variations, $\sqrt{\Sigma(\bar{S}-S)^{2}} / \bar{S}$. In the asymptotic theoretical limit, the modulation index of broad-band variations of a point source (a source subtending an angle much smaller than the scattering angle) reaches unity at a "critical frequency". Above and below this frequency the modulation index drops.

We have calculated the modulation index for each of the observations and present the results in Table 3. However, during the period of slow modulations the "modulation index" calculated during one run has little meaning, as typically only one scintle is seen per observation. We therefore also present in Table 3 a modulation index calculated over the entire period July to October for both years.

We calculate the error on the modulation index from Monte Carlo simulations. We used the observations which display over eight strong intensity maxima, and selected portions of these runs, to simulate the epochs where the variations are slower and we have few scintles. We calculate both an estimate of the scatter on calculated modulation indices, and an estimate of the underestimation that is caused by observing fewer scintles (Narayan et al. 1989; Deshpande \& Nityananda 1990). For an observation of $N$ high maxima ("scintles"), the scatter on the calculated modulation index is given by $\sim 0.3 \mathrm{~m} / \sqrt{N}$, and the underestimation of the modulation index is typically around half this. The error on the calculated mean flux density $S$ is found to be $\sim S / 6 N$, or $2 \%$, whichever is the larger.

For the period of short observations we have calculated the mean flux densities and modulation indices. These are shown at 


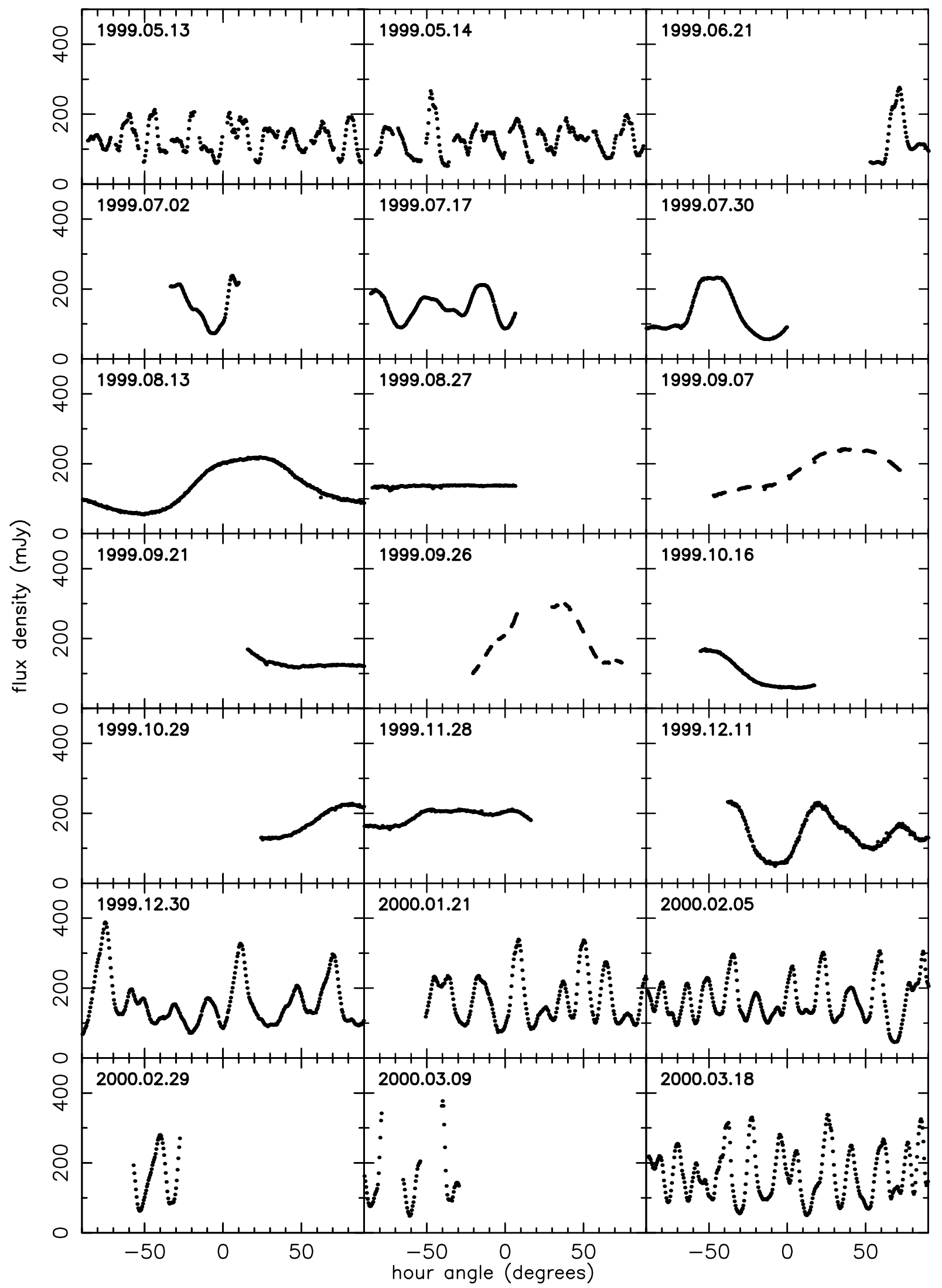

Fig. 2. Two years of monitoring observations of J1819+3845 at $5 \mathrm{GHz}$. The first page covers 11 months, the second page 15 months. The annual change in the timescale of the modulations is clearly apparent.The horizontal axis shows the Hour Angle of the source at the WSRT, so that each panel covers to 12 hours. 


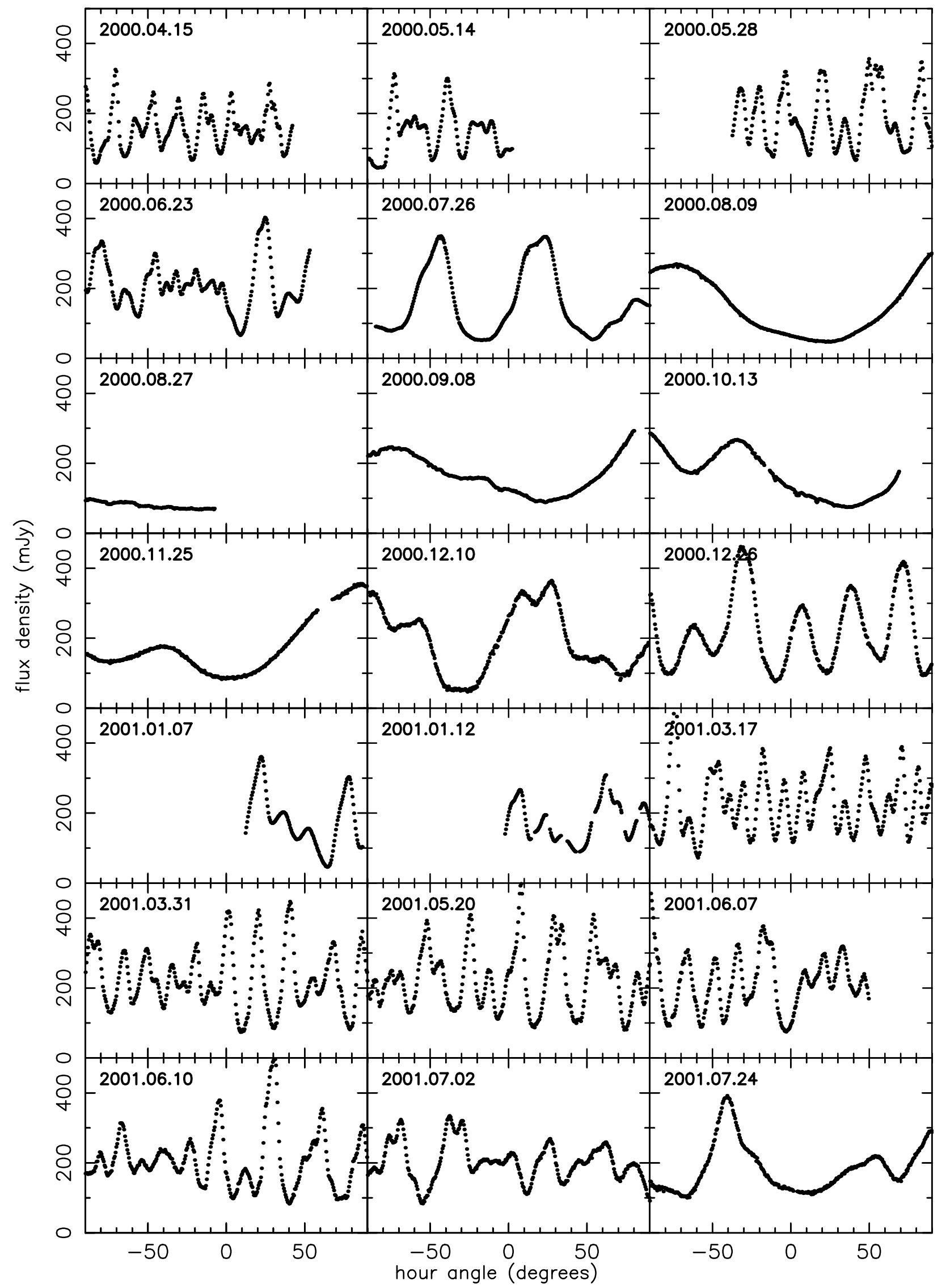

Fig. 2. continued. 


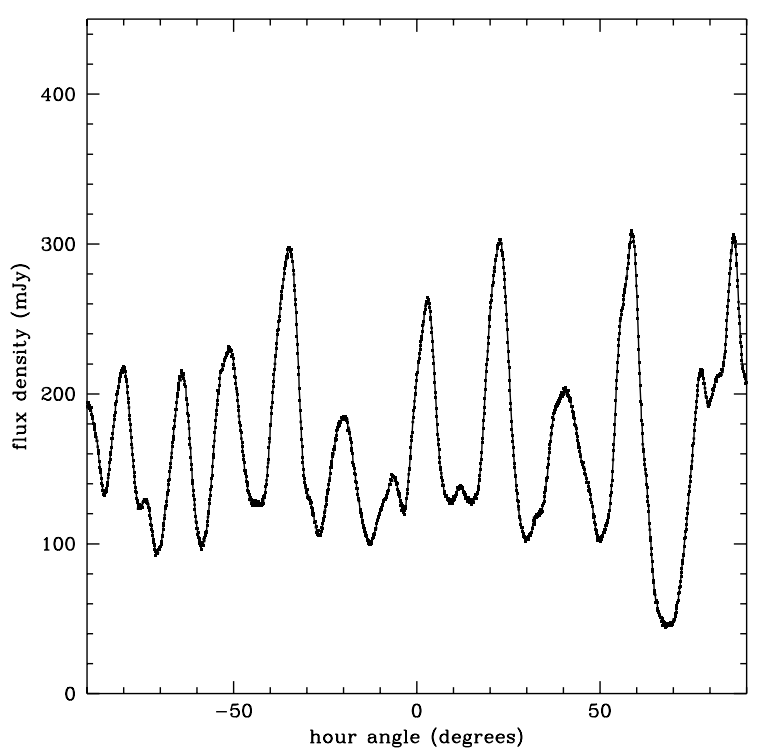

Fig. 3. The light curve at $30 \mathrm{~s}$ resolution from 5 February 2000.

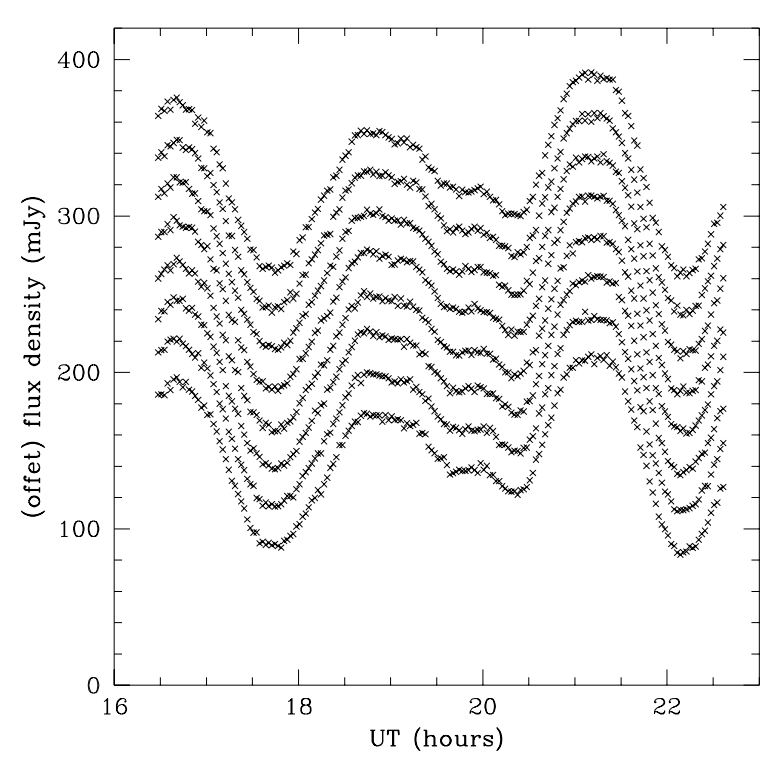

Fig. 4. The light curves from 17 July 1999 at 120 s resolution showing all eight $10 \mathrm{MHz}$ bands. The lowest light curve corresponds to a central frequency of $4.839 \mathrm{GHz}$. The other bands, each $10 \mathrm{MHz}$ higher in frequency than the previous, are displayed with an offset interval of $25 \mathrm{mJy}$.

the end of Table 3. As the observed flux density is not normally distributed, we again estimate the error on the calculated quantities by use of Monte Carlo simulations. This time we drew a number of flux densities randomly from an observation with many variations. We conclude that the error on the calculated modulation index, $m$, after $N$ observations, can be expressed as $\sim 2 m / 3 \sqrt{N}$. The error on the calculated mean $\bar{S}$ can be expressed as $\sim \bar{S} /(3 \sqrt{N})$.

From this analysis we conclude that the modulation indices at $5 \mathrm{GHz}$ are not statistically distinguishable throughout the observing period (Fig. 6). They remain constant at $\approx 0.40$.

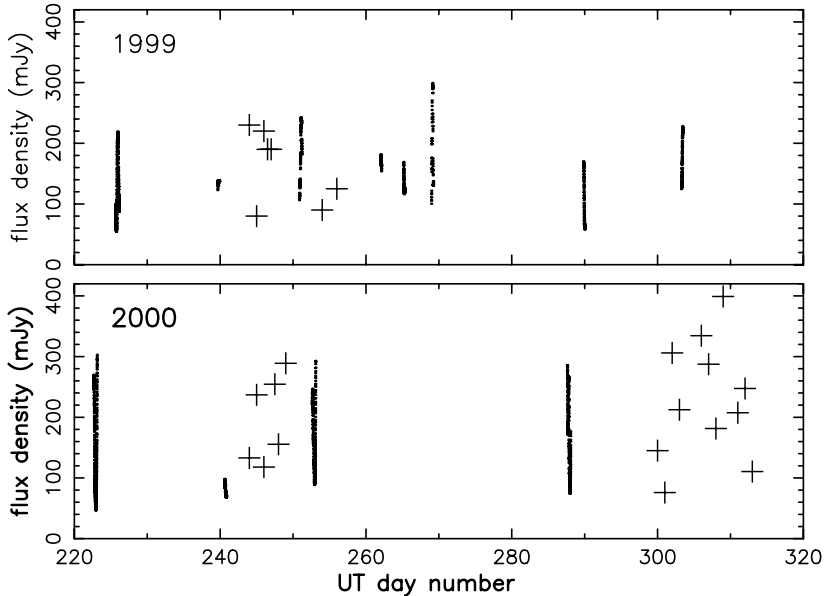

Fig. 5. Observations in August through October in 1999 (top panel) and 2000 (bottom panel). In the 6-hour observation on 27 August 1999 (day 239), the source appears to maintain a near-steady flux density: but the variations have not stopped, merely slowed down.

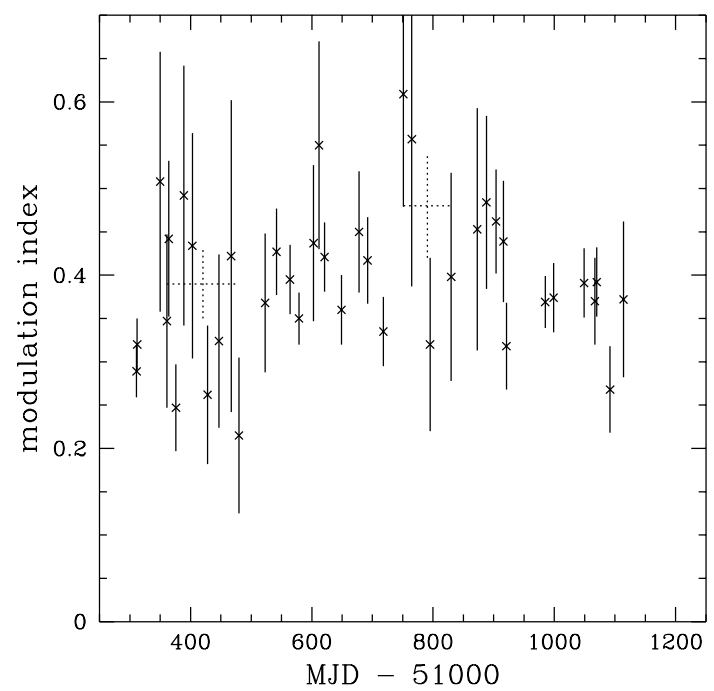

Fig. 6. Modulation index as a function of observing date. The mean flux densities used in this plot correspond to the mean measured during the respective observation. The two dotted crosses mark the modulation index calculated using all the observations in the slow period between July and October. Our estimation of the errors, given by the vertical bars, is explained in the text.

\subsection{Timescales of variation}

The timescale of the variations can be defined in a number of ways. The timescale used in Paper I was defined by use of the structure function, as this is the quantity easily related to the theoretical studies of scintillation. However this measure is impossible to compute with less than one observed scintle. With very few observed modulations any measure of the timescale will obviously be dominated by statistical error. Therefore we have used independent ways of estimating the timescale in an attempt to minimize the effect of this on our conclusions. We describe these three ways of calculating the timescale below, and present the results in Cols. 8 to 11 of Table 3 . 
Table 3. Derived quantities: Col. 5 is the number of scintles observed, $N$. Columns 6 and 7 list the modulation index and associated error. Columns 8, 9, 11 show the derived timescales: using the structure function and derived modulation index, using $m=0.4$, and an estimation based on the average time between extrema, respectively.

\begin{tabular}{|c|c|c|c|c|c|c|c|c|c|c|}
\hline Date & $\begin{array}{l}\text { mean } \\
(\mathrm{mJy})\end{array}$ & $\begin{array}{r}\min \\
(\mathrm{mJy})\end{array}$ & $\begin{array}{r}\max \\
(\mathrm{mJy})\end{array}$ & $N$ & $m$ & $\sigma_{m}$ & $\begin{array}{r}t \\
(\operatorname{mins})\end{array}$ & $\begin{array}{r}t^{\prime} \\
(\operatorname{mins})\end{array}$ & $\begin{array}{r}\sigma_{t} \\
\text { (mins) }\end{array}$ & $\begin{array}{r}t^{\star} \\
(\operatorname{mins})\end{array}$ \\
\hline 1999-05-13 & 131.0 & 55.7 & 215.8 & 11.0 & 0.29 & 0.03 & 11.51 & 11.51 & 0.87 & 76 \\
\hline 1999-05-14 & 126.6 & 47.3 & 271.1 & 10.0 & 0.32 & 0.03 & 14.71 & 14.71 & 1.16 & 77 \\
\hline 1999-06-21 & 129.5 & 55.5 & 277.3 & 1.0 & 0.51 & 0.15 & 18.85 & 14.00 & 4.71 & 110 \\
\hline 1999-07-02 & 152.7 & 70.4 & 239.4 & 1.0 & 0.35 & 0.10 & 26.00 & 32.00 & 6.50 & 156 \\
\hline 1999-07-05 & 144.5 & 58.1 & 277.0 & 2.0 & 0.44 & 0.09 & 29.43 & 27.30 & 5.20 & 180 \\
\hline 1999-07-17 & 148.6 & 83.3 & 213.5 & 2.0 & 0.25 & 0.05 & 26.24 & 55.00 & 4.64 & 270 \\
\hline 1999-07-30 & 125.9 & 53.8 & 234.9 & 1.0 & 0.49 & 0.15 & 51.59 & 41.50 & 12.90 & 270 \\
\hline 1999-08-13 & 129.9 & 54.5 & 219.1 & 1.0 & 0.43 & 0.13 & 115.60 & 101.00 & 28.90 & 560 \\
\hline 1999-09-07 & 180.4 & 102.7 & 244.0 & 1.0 & 0.26 & 0.08 & 130.62 & 227.00 & 32.66 & 620 \\
\hline 1999-09-26 & 197.0 & 97.0 & 301.1 & 1.0 & 0.32 & 0.10 & 53.00 & 66.00 & 13.25 & 400 \\
\hline 1999-10-16 & 97.9 & 56.1 & 172.1 & 0.5 & 0.42 & 0.18 & 82.12 & 77.00 & 29.03 & 400 \\
\hline 1999-10-29 & 176.1 & 120.2 & 230.5 & 0.5 & 0.22 & 0.09 & 80.71 & 150.00 & 28.54 & 550 \\
\hline 1999-12-11 & 139.8 & 45.5 & 247.0 & 2.0 & 0.37 & 0.08 & 42.29 & 46.90 & 7.48 & 220 \\
\hline 1999-12-30 & 157.6 & 58.9 & 395.7 & 7.0 & 0.43 & 0.05 & 22.07 & 22.07 & 2.09 & 97 \\
\hline 2000-01-21 & 168.3 & 69.7 & 342.7 & 8.0 & 0.40 & 0.04 & 14.78 & 14.78 & 1.31 & 94 \\
\hline $2000-02-05$ & 166.3 & 44.3 & 308.8 & 10.0 & 0.35 & 0.03 & 14.27 & 14.27 & 1.13 & 68 \\
\hline $2000-02-29$ & 157.8 & 61.0 & 281.6 & 2.0 & 0.44 & 0.09 & 12.76 & 11.20 & 2.26 & 78 \\
\hline 2000-03-09 & 139.7 & 48.0 & 379.7 & 2.0 & 0.55 & 0.12 & 9.18 & 9.18 & 1.62 & 51 \\
\hline 2000-03-18 & 168.5 & 48.7 & 342.1 & 12.0 & 0.42 & 0.04 & 11.65 & 11.65 & 0.84 & 62 \\
\hline 2000-04-15 & 156.4 & 56.0 & 329.6 & 8.0 & 0.36 & 0.04 & 11.30 & 11.30 & 1.00 & 68 \\
\hline 2000-05-14 & 143.3 & 42.5 & 314.2 & 4.0 & 0.45 & 0.07 & 15.42 & 13.06 & 1.93 & 103 \\
\hline $2000-05-28$ & 189.1 & 65.6 & 358.7 & 7.0 & 0.42 & 0.05 & 14.54 & 14.54 & 1.37 & 78 \\
\hline 2000-06-23 & 209.4 & 64.4 & 403.4 & 7.0 & 0.34 & 0.04 & 20.67 & 20.67 & 1.95 & 76 \\
\hline 2000-07-26 & 155.7 & 50.1 & 352.2 & 2.0 & 0.61 & 0.13 & 46.50 & 46.50 & 8.22 & 256 \\
\hline 2000-08-09 & 147.4 & 44.8 & 303.3 & 1.0 & 0.56 & 0.17 & 127.79 & 87.00 & 31.95 & 720 \\
\hline 2000-09-08 & 168.0 & 86.1 & 295.3 & 1.0 & 0.32 & 0.10 & 113.85 & 165.00 & 28.46 & 720 \\
\hline 2000-10-13 & 163.2 & 72.2 & 287.1 & 1.0 & 0.40 & 0.12 & 124.00 & 124.00 & 31.00 & 400 \\
\hline 2000-11-25 & 171.0 & 78.3 & 361.2 & 1.0 & 0.45 & 0.14 & 139.50 & 120.50 & 34.88 & 370 \\
\hline $2000-12-10$ & 190.1 & 32.6 & 376.3 & 2.0 & 0.48 & 0.10 & 68.72 & 68.72 & 12.15 & 290 \\
\hline 2000-12-26 & 217.7 & 75.9 & 461.7 & 5.0 & 0.46 & 0.06 & 27.66 & 27.66 & 3.09 & 130 \\
\hline 2001-01-07 & 179.3 & 45.3 & 362.8 & 4.0 & 0.44 & 0.07 & 21.98 & 19.00 & 2.75 & 75 \\
\hline 2001-01-12 & 177.8 & 87.5 & 309.4 & 4.0 & 0.32 & 0.05 & 22.11 & 39.00 & 2.76 & 105 \\
\hline 2001-03-17 & 228.3 & 66.4 & 527.6 & 12.0 & 0.37 & 0.03 & 11.62 & 11.62 & 0.84 & 50 \\
\hline 2001-03-31 & 226.5 & 66.2 & 453.0 & 10.0 & 0.37 & 0.04 & 13.64 & 13.64 & 1.08 & 72 \\
\hline 2001-05-20 & 223.1 & 69.9 & 503.6 & 8.0 & 0.39 & 0.04 & 15.54 & 15.54 & 1.37 & 110 \\
\hline 2001-06-07 & 216.1 & 71.5 & 483.3 & 6.0 & 0.37 & 0.05 & 16.39 & 16.39 & 1.67 & 80 \\
\hline 2001-06-10 & 207.7 & 79.4 & 507.7 & 8.0 & 0.39 & 0.04 & 17.45 & 17.45 & 1.54 & 120 \\
\hline 2001-07-02 & 202.1 & 79.2 & 341.8 & 3.0 & 0.27 & 0.05 & 23.78 & 51.00 & 3.43 & 180 \\
\hline 2001-07-24 & 183.2 & 95.0 & 394.5 & 1.5 & 0.37 & 0.09 & 52.33 & 59.00 & 10.68 & 480 \\
\hline \multicolumn{11}{|c|}{ Characterization of short observations ( $N=$ number of observations $)$} \\
\hline 1999.09.01-09.19 & 159 & 83.0 & 234.0 & $(10)$ & 0.32 & 0.07 & & & & \\
\hline $2000.08 .31-09.05$ & 198 & 118.0 & 289.0 & $(6)$ & 0.36 & 0.10 & & & & \\
\hline 2000.10.25-11.07 & 228 & 76.0 & 399.0 & (11) & 0.43 & 0.08 & & & & \\
\hline \multicolumn{11}{|c|}{ Combined data from slow season } \\
\hline 1999.07-10 & 148 & 53.8 & 277.8 & & 0.39 & 0.04 & & & & \\
\hline $2000.07-10$ & 158 & 44.8 & 352.2 & & 0.48 & 0.06 & & & & \\
\hline
\end{tabular}

For our regularly sampled light curves $f(i), i=1,2 \ldots n$, the first order structure function is estimated as

$$
D_{f}^{1}(k)=\frac{1}{\sum w(i) w(i+k)} \sum_{i=1}^{n} w(i) w(i+k)[f(i+k)-f(i)]^{2}
$$

where $w(i)=1$ if the measurement exists and is otherwise zero (Simonetti et al. 1985). The structure function analysis is straightforward for those observations with a large number of modulations. In this case it can be seen that the structure function has saturated (see e.g. Fig. 7), and we can calculate the timescale as the time at the $1 / e$ of this maximum value 


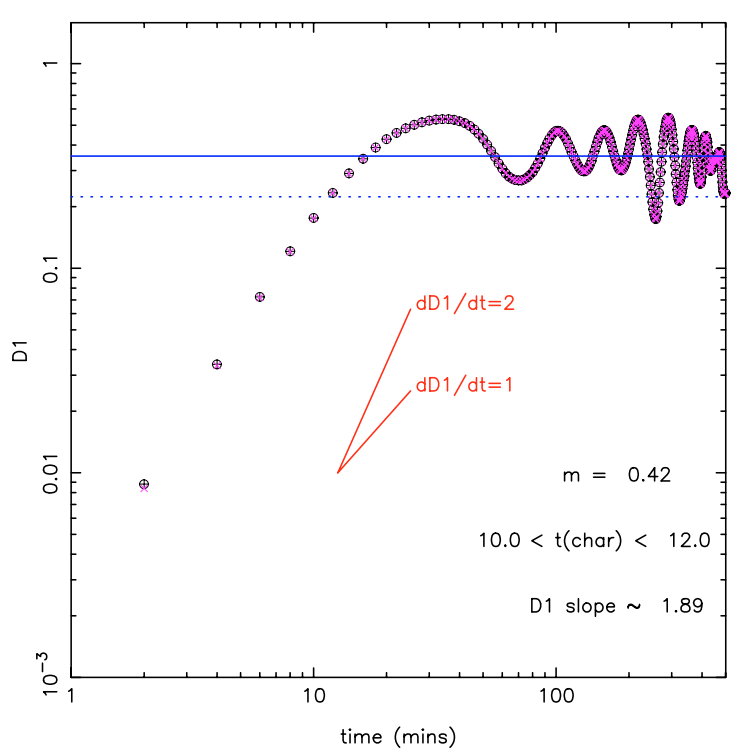

Fig. 7. A saturated structure function with observations from 18/03/2000. Crossed circles are points obtained directly from the data, and the crosses are after correction for thermal noise (see text). The error on the calculated timescale is dominated by statistical error from a finite number of observed variations, not the thermal noise correction. The solid lines indicate slopes $D^{1} \propto t^{2}$ and $t$ (see Sect. 7).

(which is $D^{1}(\max )=2 \mathrm{~m}^{2}$ ). This is given by $t$ in Col. 8 of Table 3.

Following the error analysis of Simonetti et al. (1985) the observed value $D_{f}^{1}(k)$ exceeds the true value by an additional quantity $2 \sigma_{\delta f}^{2}$, where $\sigma_{\delta f}^{2}$ is the measurement noise variance (assuming white noise with correlation timescale smaller than the smallest time interval measured). We thus assess the timescale correcting each value of $D^{1}$ with the relevant noise variance, but the correction on the calculated timescale is insignificantly small ( $\sim 1 \mathrm{~s}$ for the most rapid variations). The error on the calculated structure function $D_{f}^{1}(k)$ from this contribution is negligible. From the Monte Carlo simulations, we find that the (one sigma) error on this timescale due to statistics of finite observing period is $\sim 0.25 t / \sqrt{N}$.

For observations with few $(\leq 5)$ scintles we estimate a timescale from the structure function by assuming a mean modulation index for all epochs. By considering the modulation indices obtained in May 1999, and December 1999 through May 2000, we take a modulation index of $m=0.4$ for all epochs and estimate the timescale as the time at which the structure function (or its extrapolation) reaches $2 \mathrm{~m}^{2} / \mathrm{e}$. This modification is given by $t^{\prime}$ in Col. 9 of Table 3 .

As a final, and most direct, approach, we also estimated the timescale, $t^{\star}$, from the average time between two minima (or maxima) during the observation run. For runs with a small number of scintles, $t^{\star}$ was calculated as the average of twice the time between a minimum and maximum. We estimate errors on this process by considering the runs with $>5$ complete modulations. We calculated the $\sigma$ on the timescale as measured from each single complete modulation independently. We found that the error on the measured timescale can be estimated as $\sigma_{t^{\star}}=0.3 t^{\star} / \sqrt{ } N$, where $N$ is the complete number of modulations observed during the run. We calculated $t^{\star}$ in order to assess the reliability of our error estimates on runs with few scintles, and their possible propagation into the final results. The quantitative results obtained using $t^{\star}$ are quoted in Sect. 6 , but we note here that the results so obtained were similar to those obtained by the other estimations.

For the observations of 9 March 2000, it is not clear how many minima occur during the run, due to gaps in the observations. Considering the rapid change of flux density on this date, it is plausible that extra minima also occur during the unobserved periods. The timescale quoted is based on this assumption, the error used in the fits is based on the difference between this and the timescale calculated if only 4 minima occur during the observations. We can obtain lower limits to $t^{\star}$ from the duration of the observations during which the light curves showed little variation: 1999-08-27 (360 min), 1999-09-21 (300 min), 1999-11-28 (400 min), 2000-08-27 (400 min). This information is not included in the fitting.

\section{Detection of peculiar velocity of scattering plasma}

Figure 8 shows the changing timescale $(t)$ as a function of epoch throughout the period of observations. The yearly changes in the timescale can also be clearly seen directly in the data (Fig. 2).

A change in the timescale of the scintillations with a year periodicity must be due to the changing speed at which the Earth cuts through the scintillation pattern projected onto the Solar System, as we move around the Sun. The velocity of the scintillation pattern, as viewed by a stationary observer on Earth, is given by the relative velocity of the Earth and scattering plasma projected onto a screen perpendicular to the direction of the source, $v_{\text {Earth }}-v_{\text {plasma }}$. We note that such a model predicts no change in the modulation index.

For most extragalactic sources, the vector $v_{\text {Earth }}$ itself will provide a substantial yearly modulation in the pattern speed. This is in contrast to pulsars, which produce scintillation patterns whose observed motion is dominated by the pulsar's own velocity of typically several hundreds $\mathrm{km} \mathrm{s}^{-1}$. One exception is PSR B2016+28, which has a transverse proper motion velocity comparable to the Earth's. Gupta et al. (1994) found a variation in the scintillation timescales of pulsars, which they attributed to an annual variation caused by the Earth's motion. Unlike most extragalactic sources, J1819+3845 is not expected to show a large annual modulation in the pattern speed. This is illustrated by the dotted line in Fig. 8. J1819+3845 is just $9^{\circ}$ from the direction of Sun's motion with respect to the LSR, and $28^{\circ}$ from the ecliptic pole, so the modulation due to the Earth's motion is slight.

In order to fit the large change in timescale during the year we require that the scattering plasma itself has a velocity with respect to the solar rest frame (taken as moving at $19.7 \mathrm{~km} \mathrm{~s}^{-1}$ towards $180750,+300052$ (J2000)). The change in timescale throughout the year is assumed to be entirely due to changes in our velocity through an isotropic scintillation pattern. Thus we assume that the size of the scintles, angular size of the source, 


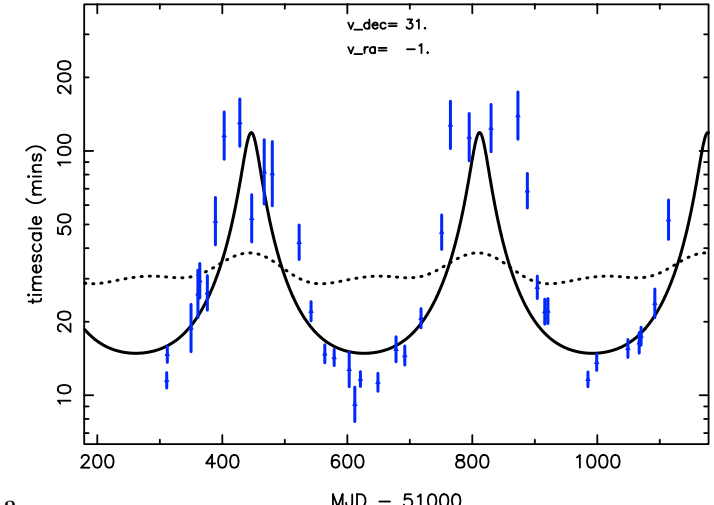

a.

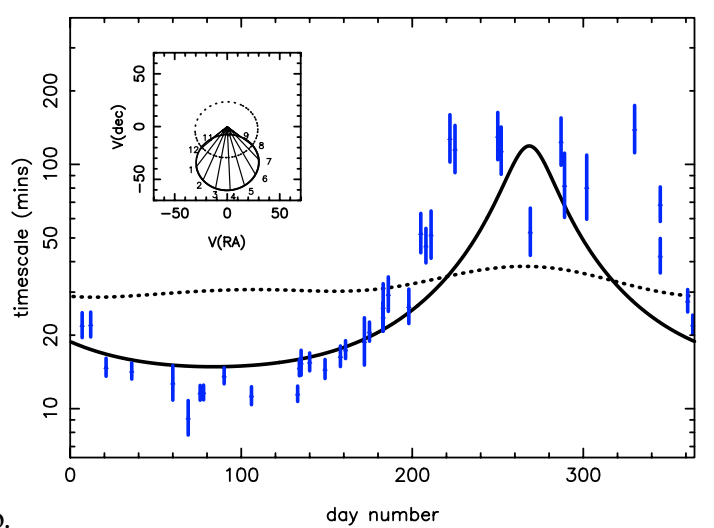

Fig. 8. The timescales, $t$, (logarithmic scale) of the variations as a function of observation date. The dotted line corresponds to the prediction if the scattering plasma has no peculiar velocity. The solid line gives the best fit to the data (using timescale estimate $t$ ) if the plasma is allowed a peculiar velocity. The lower panel b) shows the data folded on a year. The annual modulation, as well as the width of the epoch of slow modulations can be clearly seen. The corresponding monthly velocity vectors $v_{\text {Earth }}-v_{\text {plasma }}$ are shown in the ellipses: near-circular around the origin for no plasma motion, and offset for the fit shown (see also Fig. 11).

and distance to the scattering screen are constant throughout the years. However, we neither fix nor determine these values, as the change in timescale only depends on the changing projected velocity.

We probed a region of parameter space for the projected velocity of the scattering plasma between $-50 \mathrm{~km} \mathrm{~s}^{-1}$ and $+50 \mathrm{~km} \mathrm{~s}^{-1}$ in both RA and declination, and calculated the best fit. The best fits were clearly constrained in this space, and the best weighted $\chi^{2}$ fit to the calculated timescales is given by the solid line in Fig. 8. It shows that the change in timescale can be qualitatively explained by a velocity of the scattering plasma of $\sim 30 \mathrm{~km} \mathrm{~s}^{-1}$. The model allows the timescale to be normalised according to the best-fit. The ellipses in Fig. 8b correspond to the projected velocities, for the cases of no peculiar plasma velocity (dotted line) and a plasma velocity corresponding to the fit shown (solid line). The velocity vectors (using the fitted plasma velocity) are also shown for the beginning of each month.

Although the model qualitatively describes the data, with an annual period of long modulations, it is clear that the fit is poor (reduced $\chi^{2}=9.1$ for $t$; goodness of fit parameter $Q=10^{-38}$ ). The fit is similar using the other timescale estimates. The period of very slow modulations extends for a much longer time than is allowed by this simple model. Nor is the sharp reduction in timescale in December, or sharp increase in timescale in July/August each year replicated by this model. Furthermore the fitted plasma velocity does not agree well with that measured from a delay experiment (Paper II). However, a peculiar velocity of the scattering plasma is the only possible explanation for this annual variation. In the next section we consider further refinements and additions to the model which can explain the long period over which slower modulations are observed.

\section{Detection of source structure/ anisotropic scattering medium}

In the preceding section we assumed that the scintillation pattern was isotropic. Anisotropic scintles could be produced by either an extended anisotropic source or an anisotropic scattering medium. An anisotropic scintillation pattern would leave a distinctive trace in the form of an annual variation of the timescale. This would happen as the Earth moves around the Sun, and we cut the pattern at varying angles through the anisotropic pattern, thereby introducing an annual variation in the timescale of modulations.

In the absence of any peculiar velocity of the scattering plasma, the Earth would cut through the scintillation pattern of J1819+3845 at the same angle, but moving in different directions, approximately every six months. Thus, for this source in the absence of any peculiar scattering plasma, the lengthening of the timescale would occur about every six months. It is therefore clear that source structure cannot be the only explanation of the observed changes in timescale.

With the addition of a peculiar velocity of the scattering medium, however, the periods of slow modulations no longer occur every six months. It is therefore possible that both a peculiar plasma velocity and source structure combine to produce the observed annual variation in timescale.

We modelled an anisotropy in the scintillation pattern as an ellipsoid, introducing two new parameters: the position angle and axial ratio of the scintles. Using these extra parameters we again performed a $\chi^{2}$ minimization over a wide range of parameter space. The resulting best fits are significantly improved over the fits without the anisotropy term (reduced $\chi^{2}$ is 1.5 for $t$; 5.2 for $t^{\star} ; 7.1$ for $t^{\prime}$ ). Figure 9 shows the probability contours. Although the fit was improved, good solutions were available over a range of parameters. It was found that the position angle and $v_{\mathrm{dec}}$ were relatively well-constrained, but the axial ratio and $v_{\mathrm{RA}}$ were poorly constrained.

In Paper II we directly measured the velocity of the scattering plasma using the time delay of signals arriving at two telescopes: $v_{\mathrm{RA}}=-32.5 \pm 0.5 \mathrm{~km} \mathrm{~s}^{-1} ; v_{\mathrm{dec}}=$ $+15.5 \pm 1 \mathrm{~km} \mathrm{~s}^{-11}$. This result lies within the narrow region

\footnotetext{
${ }^{1}$ Note added in proof: We have since realised that full consideration of anisotropy in the two-telescope experiment is also needed. The effect of this is that the velocity is not as well constrained as given here, but the main conclusions are not significantly altered.
} 

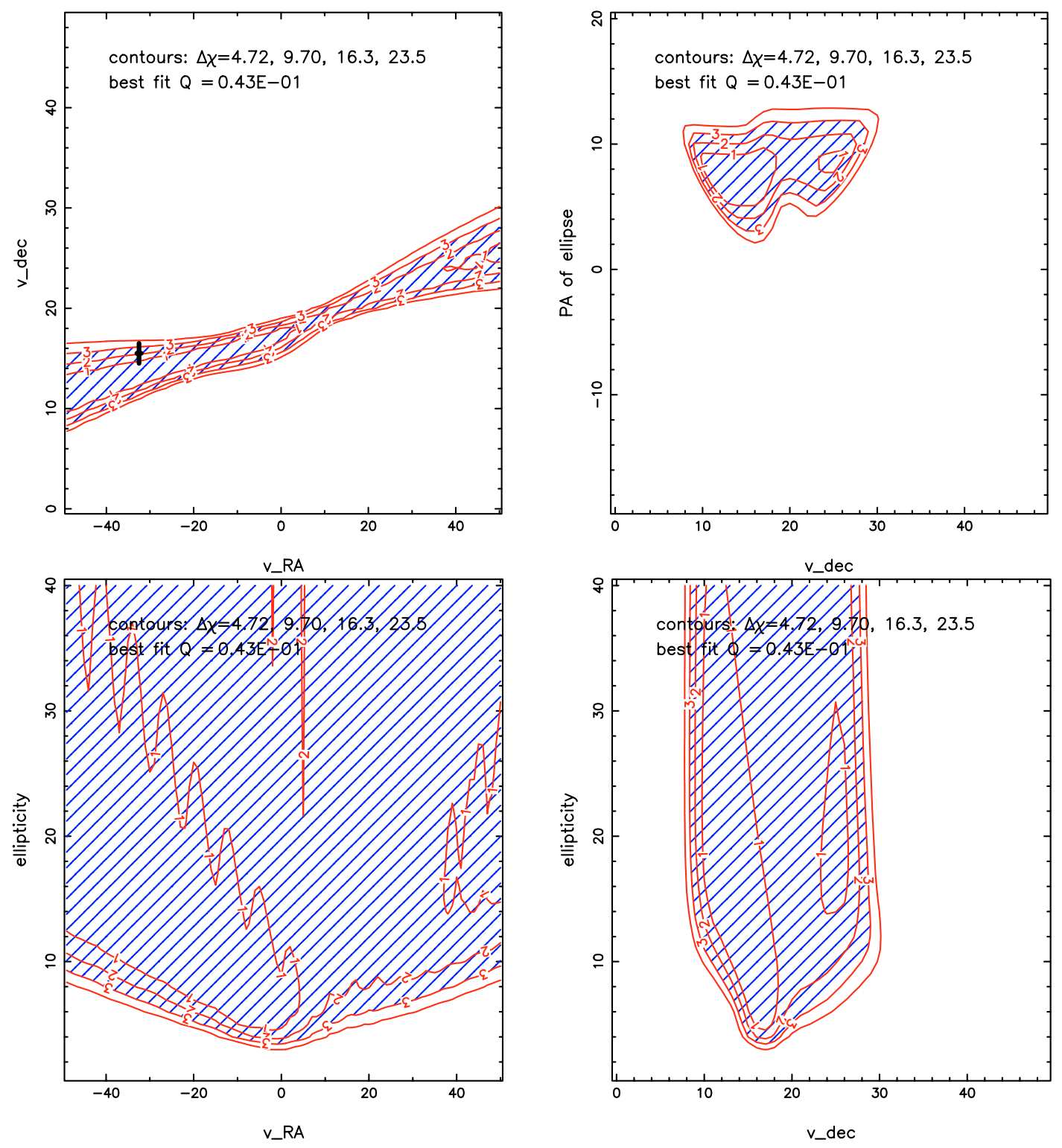

Fig. 9. The probability distributions based on $\chi^{2}$-minimization fits, using $t$. For each plot the probabilities are calculated over the ranges shown in the other plot. The contours correspond to $68.3,95.4,99.73$ and $99.99 \%$ certainties for a normal distribution of errors. The vertical line in the first panel represents the velocity and its errors derived in Paper II. Note that in these plots PA is defined as E through N.

of permissible $v_{\mathrm{RA}}-v_{\mathrm{dec}}$ space determined from these yearly monitoring observations (see Fig. 9). Constraining the velocity to be within the 3- $\sigma$ errors of this measurement, we re-ran the fit and determined a probability profile for the shape and position of the ellipse. This is shown in Fig. 10a, and the results are listed in Table 4. The best fit (at $v_{\mathrm{RA}}=-33.5 \mathrm{~km} \mathrm{~s}^{-1}$; $\left.v_{\mathrm{dec}}=+13.5 \mathrm{~km} \mathrm{~s}^{-1}\right)$ has axial ratio $14_{(+>30)}^{-8}$ at position angle $83^{\circ} \pm 4^{\circ}(\mathrm{N}$ through $\mathrm{E})$. Use of the timescales obtained from the peak separation gives a similar result, although the formal fit is very poor.

The introduction of two more parameters has clearly improved the fit sufficiently to justify their use, as can be directly seen in Fig. 10. We also note that the dates on which the observations display very little variation over 6 hours, and which therefore had no calculated timescale and were not included in the fit, occur during the periods of predicted slow modulations.
Table 4. Results of fitting, using $v_{\mathrm{RA}}=-32.5 \pm 1.5 \mathrm{~km} \mathrm{~s}^{-1} ; v_{\mathrm{dec}}=$ $15.5 \pm 3 \mathrm{~km} \mathrm{~s}^{-1}$. Errors are 3- $\sigma$.

\begin{tabular}{lllll}
\hline \hline & axial ratio, $\epsilon$ & position angle & $\chi^{2}$ & $Q$ \\
\hline$t$ & $14_{+(>30)}^{-8}$ & $83 \pm 4$ & 1.5 & $4 \mathrm{e}-2$ \\
$t^{\star}$ & $11_{+10}^{-4}$ & $88 \pm 4$ & 5.6 & $2 \mathrm{e}-23$ \\
$t^{\prime}$ & $31_{+(>10)}^{-10}$ & $83 \pm 4$ & 7.2 & $1 \mathrm{e}-32$ \\
\hline
\end{tabular}

The fit is illustrated in an illuminating way in Fig. 12 which shows the data compressed by the timescales predicted from the model, such that the effects of plasma motion and anisotropy are removed. After this timescale adjustment, the similarity of the modulations throughout the two years can be clearly seen. The timescale of the variations now looks similar 
(a)
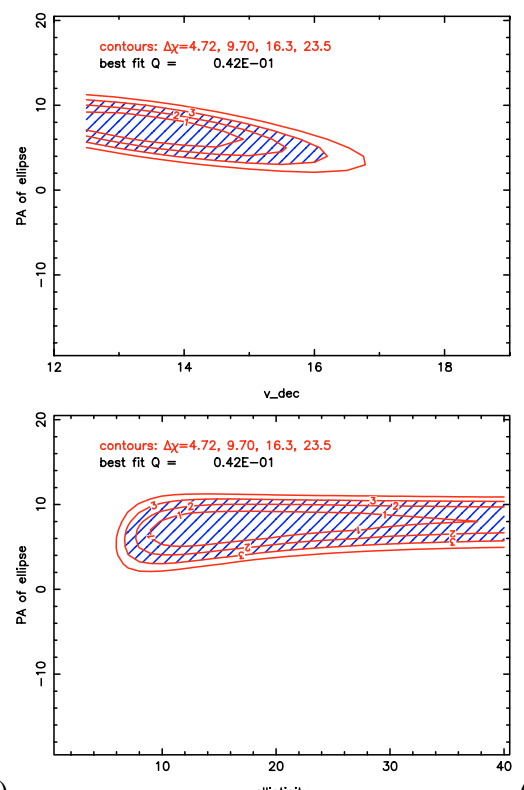

Fig. 10. Fitting constrained by the plasma velocity (and error) from Paper II. Timescale estimate $t$ has been used. a) The probability distributions based on $\chi^{2}$ fits, showing the constraints placed on the axial ratio and position angle of the anisotropy of the scintles. b) The resulting best fit to the timescales with epoch.

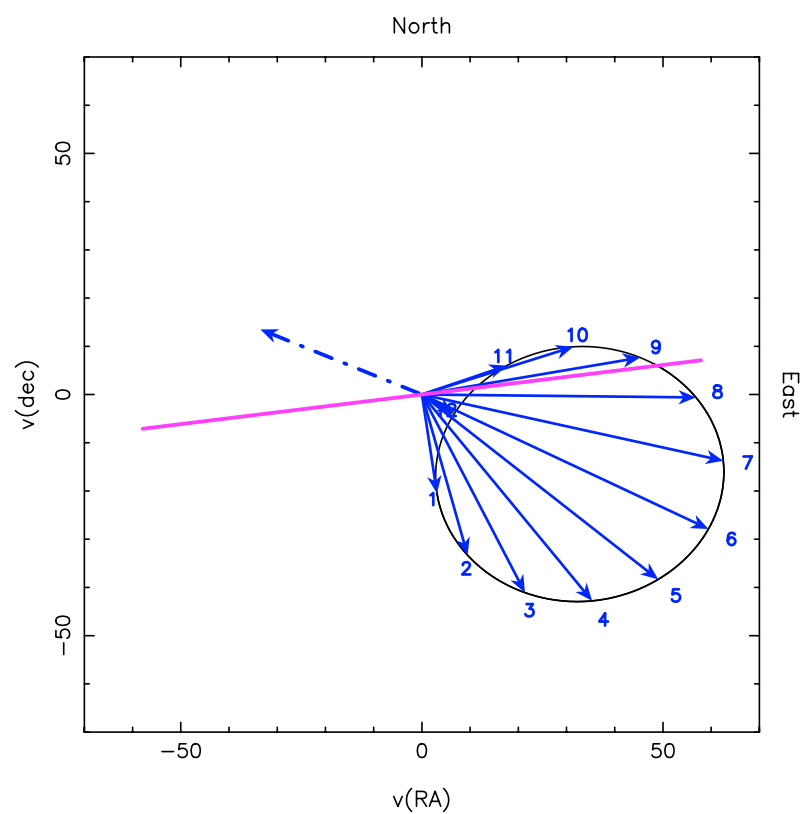

Fig. 11. The velocity vectors on the first of the month for the best fit model, using the constraints from Paper II. The velocity vectors are the velocity of the Earth through the scintillation pattern $\left(v_{\text {(Earth) }}-v_{\text {(plasma) }}\right)$, as seen from the source. The dashed-dotted line shows the projected plasma velocity ( $\left.v_{\text {(plasma) }}\right)$. In order to illustrate the combined effects of the velocity and the anisotropy we also present, on the same plot, the position angle of the anisotropy as seen from the source (solid line).

throughout the period, indicating the model fit was suitable. The nature of the variations is, by eye, also similar throughout the observing period: there is no indication of big changes in the "jaggedness", or the number and frequency of big excursions. What is clear from the figure however, is the increasing flux density of the brightest peaks in the light curves. The minimum flux densities seem to have changed little, showing at most a slight increase. This information is also reflected in Table 3 (Cols. 3 and 4). As was shown in Fig. 6 there has been no change of rms variations over time. The change in appearance of the light curves over time, in particular the increase in high maxima, is due to the increasing flux density of the source (see Sect. 8).

One manner of producing the ellipsoidal scintles is by an ellipsoidal source, as the scintillation intensity pattern produced by an extended source is the convolution of the scintillation pattern of a point source with the brightness distribution of the source itself. In the strong, refractive regime a resolved source (source size $\theta_{\mathrm{S}}>\theta_{\text {scatt }}$ ) is observed to have a timescale of modulation larger than that of a point source as $\tau \propto \theta_{\mathrm{S}} / \theta_{\text {scatt }}$ (Narayan 1992), where $\theta_{\mathrm{S}}$ is the width of the source. If we assume that the anisotropy is then due to an ellipsoidal source undergoing strong scattering, we may equate the ellipsoid calculated above with that of the source itself.

We note that we are observing near the critical frequency where the asymptotic theory will not hold to great accuracy. However, as the asymptotic theory from the other side of the critical frequency, in the weak scattering regime, predicts $\tau \propto$ $\theta_{\mathrm{S}}^{7 / 6}$, we take $\tau \propto \theta_{\mathrm{S}}$ as a reasonable approximation. The second complication is that to apply the theory as simply as we have done at least requires the source to be larger than the scattering disk in all directions. We believe however this is a reasonable assumption, as a source that was significantly smaller than the scattering disk in one direction should be expected to display 


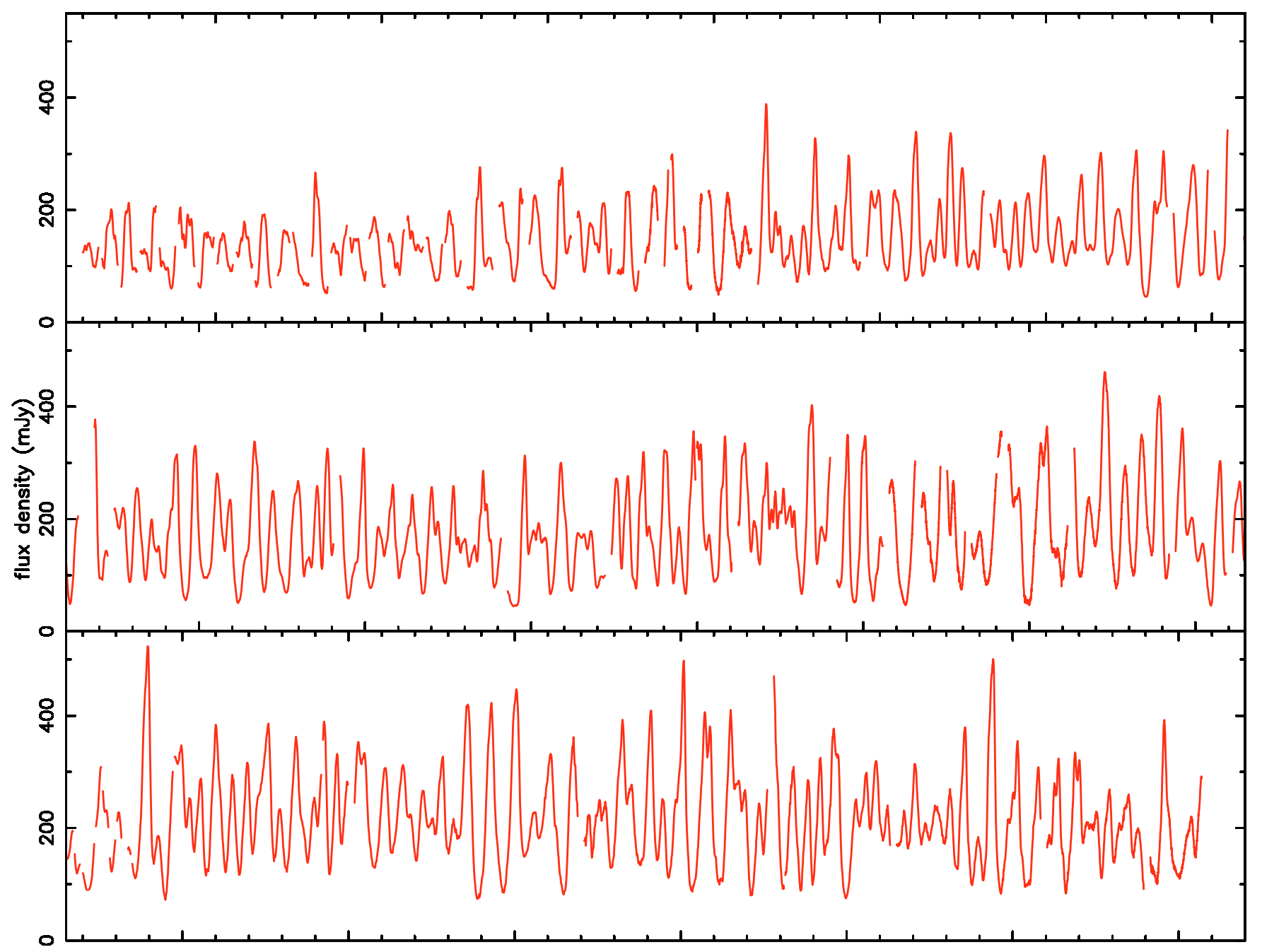

Fig. 12. The data adjusted by the timescales calculated from the best fit model. The timescale of the fluctuations is now constant. The apparent change in depth of the modulations is an effect of an increase in source brightness: the rms of the intensity fluctuations about the mean does not change significantly over the years (see Fig. 6).

different characteristics of scintillations as the velocity vector cuts across its minor axis. At this time it should display more of the second branch, or diffractive, scintillations. There is no clear evidence for such a change in character, such as a second, faster, timescale of modulation. Thus for the anisotropy caused by an anisotropic source, in so far the choice of ellipsoidal model is a good representation, the values obtained (Table 4) are expected to be close to the anisotropy of the source itself.

An anisotropy in the scatterer could produce an effect similar to the one described above for an anisotropic source. Anisotropy in the scattering medium is generally expected, and has been diagnosed by elongated scatter-broadened images, with axial ratios of up to 3 found through strongly scattering regions of the Galaxy (e.g. Lo et al. 1993; Frail et al. 1994; Wilkinson et al. 1994; Trotter et al. 1998), and axial ratios $>5$ in the solar wind (e.g. Narayan et al. 1989; Armstrong et al. 1990). Compressible MHD turbulent models have been developed in which the magnetic field gives rise to an elongation of the electron-density fluctuations, with potentially very large axial ratios (Lithwick \& Goldreich 2001). The effect of this anisotropic medium on the scintillation observables is greatest when the screen is thin, the magnetic field perpendicular to the line of sight and with little change of magnetic field direction in the scatterer. In order to produce the $>6: 1$ axial ratio which would be required for $\mathrm{J} 1819+3845$, we see from Chandran \& Backer (2002) that the magnetic field in the plane of the scattering screen must rotate through less than $\pi / 4$ radians through the screen. This implies that if anisotropy in the plasma density structure causes the observed anisotropic scintles, the magnetic field must be well ordered through the scattering screen (i.e. along line of sight). The apparent $>6: 1$ axial ratio required is somewhat unusual for high galactic latitudes, but may be related to the unusual nature, and thinness of the screen.

\section{Grand structure functions}

In order to attempt to distinguish between the possibilities of source or plasma anisotropy, to get clues about the depth of the scattering plasma, we constructed grand structure functions $\left\langle D^{1}(\tau)\right\rangle$. For this purpose we took both the mean calculated from the individual observations, and the mean obtained by a linear fit to all the observations (Sect. 8). The data was 
scaled in time according to the solutions from Sect. 6. The errors were calculated for each point directly from all contributing data, weighted according to the number of points (after time scaling) that contribute.

Grand structure functions were calculated for all the data, and over subsets of interest, including in and across the direction of the elongation found above, and the individual years. We did not find any very significant difference in the structure functions of these sub-sets. We show in Fig. 13 the grand structure function obtained using the entire data set.

The slope of the structure function before reaching saturation is $1.82 \pm 0.05$ (i.e. $\left\langle D^{1}(\tau)\right\rangle \propto \tau^{1.82}$ ). This should be compared with $\left(\left\langle D^{1}(\tau)\right\rangle \sim \propto \tau\right)$ for classical IDV sources found by Simonetti et al. (1985). Blandford et al. (1986) interpreted their result as due to scattering in a thick screen, which they showed was able to reproduce the $D^{1} \propto \tau$ result, unlike thin scattering screens which give $D^{1} \propto \tau^{2}$.

On the basis of the slope of the structure function, it is therefore likely that the scattering in $\mathrm{J} 1819+3845$ is predominantly in a relatively thin screen (thickness $\ll$ distance). An inaccurate model for the annual timescale variations would decrease the slope of $\langle D\rangle$, but in the individual runs we did not observe structure function slopes greater that 1.9. We also note that the grand structure function overshoots its saturation value. We consider the origin of these features.

The $D \propto \tau^{1.82}$ relation could potentially be explained if the screen, whilst thin, was allowed some finite thickness. We note that $D \propto \tau^{2}$ for $\beta=4$, (where $\beta$ is the index of the power spectrum of the density fluctuations) and shallower power spectra (e.g. Kolmogorov) give rise to structure functions which rise more rapidly. Therefore shallower power spectra cannot account for the observations (Romani et al. 1986, Fig. 7).

An anisotropic scatterer could produce a structure function which overshoots its saturation value (Rickett 2001). In this case we should expect this to clearly show up as a difference in the structure functions when moving across, and along the direction of anisotropy. We see the overshoot when considering the entire dataset, and no strong evidence for a difference between the structure functions when sampling the scintles perpendicular and parallel to the elongation. We conclude that preliminary analysis does not indicate plasma anisotropy, however this result is tentative because of the small number of scintles which contribute to the GSF parallel to the elongation. Source elongation/structure and scattering plasma anisotropy may be distinguishable by more sophisticated means (Rickett et al., in prep.).

\section{The stability and structure of the source}

\subsection{Source flux density changes}

The source has apparently undergone a change in mean flux density during the two years of monitoring. Figure 14 shows the mean flux densities, with the error on them calculated as in Sect. 4.1. Fitting a linear rate of change (using a weighted least squares fit) gives a rate of change of intensity as $\sim 25 \%$ per year. A line with zero intensity change is a much poorer fit to the data. The change in flux density is also clearly seen in the distribution of observed flux densities (Fig. 14b). The mean flux densities per year (data now sampled according to the timescale fit) are $137 \mathrm{mJy}$ (1999.8), $172 \mathrm{mJy}$ (2000.5), and $215 \mathrm{mJy}$ (2001.3).

It is possible that the source became brighter at $5 \mathrm{GHz}$ in outbursts at certain epochs: in particular around February 2001 (MJD 51900). However, because of the extremity of the variations and the number of scintles observed per run, we cannot distinguish this possibility from a steady brightening.

\subsection{Source structure, and (lack of) changes}

We have no evidence for structure on arcsecond or arcminute scales in this source. From the radio spectrum (Paper I) we do not expect any such structures to contribute more than a few mJy. We have looked for evidence of extended structure by mapping several 12 hour runs. We removed $\mathrm{J} 1819+3845$ by amplitude self-calibrating the whole run on the mean flux density, and then looked for sources left in the field (which would now themselves appear variable). There are a few faint compact sources more than 1 arcminute away, which have been seen also at $1.4 \mathrm{GHz}$. We find no evidence for any extended structure associated with $\mathrm{J} 1819+3845$ to the level of $0.1 \mathrm{mJy}$ for structure with scales 5-10 arcsec.

From Sect. 6 we concluded that the scintle had an elongated structure, but that this could be due to screen anisotropy and/or source structure. If the anisotropy is due to source structure it has been very stable, and by comparison of results from two year periods, we conclude that it has changed position angle by less than $10^{\circ}$ in two years.

Further information on the source structure can be gained from the probability distribution function (PDF) of its flux density. A point-like source has a very skew probability distribution function of the intensities, and a very jagged appearance of the time series. This is both expected in theory and seen in observations of pulsars (e.g. Rickett et al. 2000). The smoothness of the time series - the quasi-oscillatory appearance - indicates that the source is resolved on the scale of a Fresnel zone at the scattering screen.

There is also an indication of asymmetry in the source brightness. The falling edges of the flux density variations exhibit a more rapid change than the rising edges (de Bruyn \& Dennett-Thorpe 2001). Initially a puzzling result, because there was no evidence of a change in sense over the year, this result can be explained with the scattering plasma velocity. The scintillation pattern is always cut in the same sense, and the Western end of the source is brighter than the Eastern. In a future paper we will return in more detail to the scintle asymmetry.

We also use the PDF to look for changes in source structure over the observation period. We present the PDF for the entire data (Fig. 14b), where the data has been sampled according to the theoretical timescale from the model fit in Sect. 6. The increase in brightness over the years is obvious. Normalizing on the mean for each year (or the linear change calculated above), and further increasing the sampling rate to produce independent points (i.e. only one point in the calculated timescale is sampled) allows us to calculate statistical measures of difference. Years 1999 and 2001 are statistically indistinguishable 


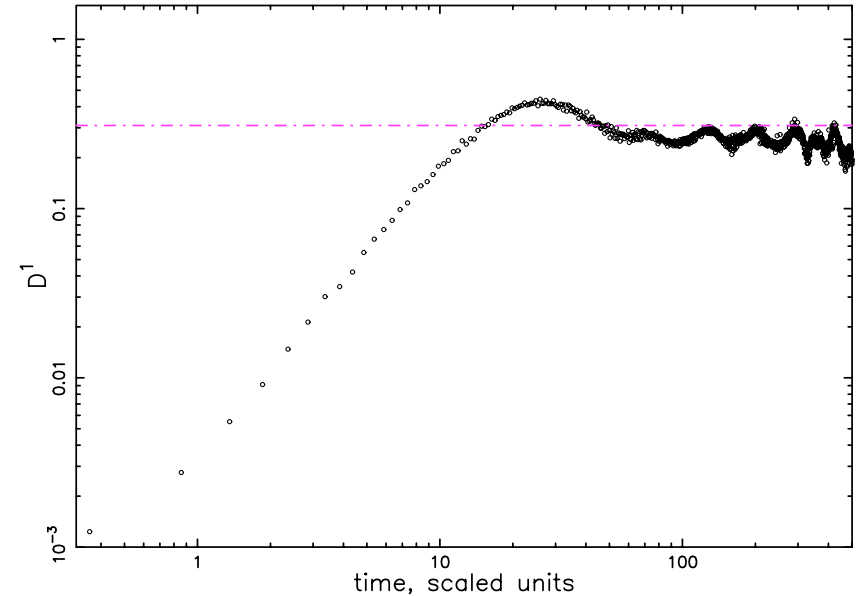

Fig. 13. Grand structure function, containing all the data, resampled according to the best fit from Sect. 6. The dashed line corresponds to the saturation value calculated from the modulation index calculated from all (resampled) data.

by the KS test ( $>r 99 \%$ certainty). However year 2000 has a $20 \%$ probability (KS test) that the distribution is similar to the other two purely by chance. This is primarily due to the higher dispersion about the mean $(\sigma=0.38,0.44,0.39$ in 1999,2000 , and 2001 respectively). From this preliminary investigation we see no evidence for change in structure.

In Paper I we considered the source to be composed of a scintillating component and a non-scintillating base component. However, the source may be a few times larger than the Fresnel scale, and all scintillating. This would also result in modulations in flux density that do not drop to zero, giving an appearance of a "base level". As previously noted, the source is expected to be resolved in order to explain the smoothness of the variations. The $50 \%$ increase in source brightness with no concomitant change in modulation index, and indeed the lack of difference in the normalized flux density PDFs over the years, is difficult to explain in the "baselevel plus small component" model. It would require that both the non-scintillating and the scintillating components increased in flux density by a similar amount, which is somewhat contrived, whereas this situation is a natural result of a resolved source that gets brighter. For this reason we now favour the picture of a resolved source, a few times the Fresnel size.

We then can interpret the modulation index in order to obtain the source size relative to the scattering zone, which we will return to in Sect. 9.

\subsection{Expansion rate}

From the modulation index alone (Fig. 6), which shows no signs of changes over the two years, we can put an upper limit on the source expansion. As we have reason to believe that the source is not much smaller than the scattering disk (above), we can directly translate the lack of decrease in modulation index to a lack of detectable source expansion. Because the scintillation pattern is a convolution of the scintillation pattern produced by a point source and the source brightness distribution,

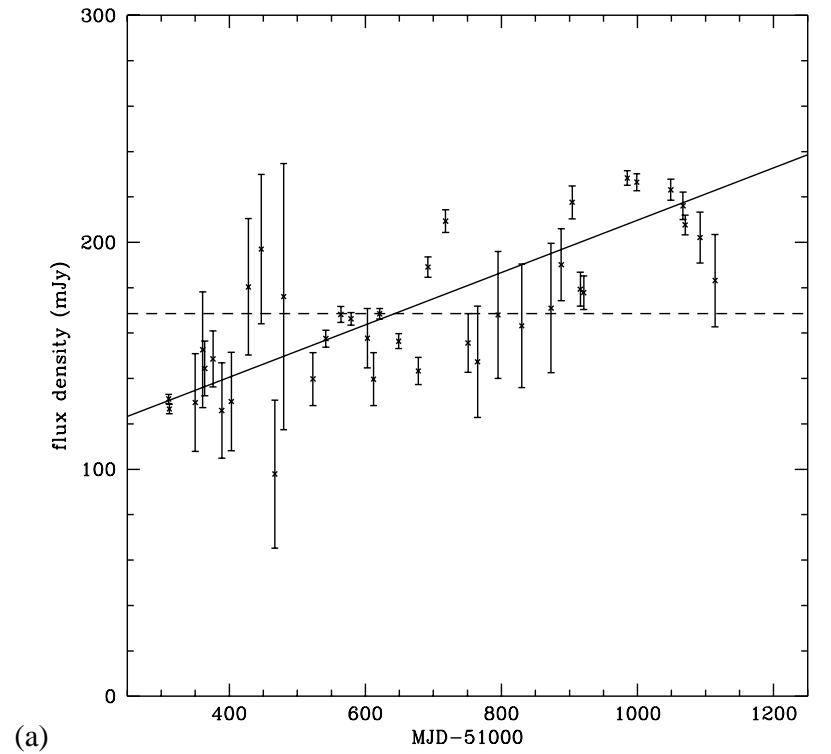

(a)

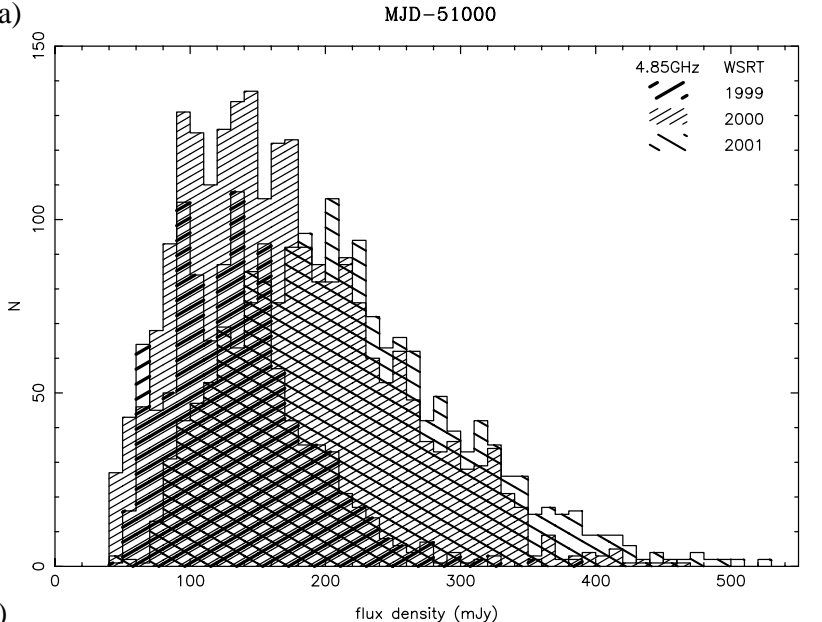

(b)

flux density (mJy)

Fig. 14. a) The mean flux density of source during the monitoring campaign. The solid line represents the best linear fit to the data; the dotted line the weighted mean. b) he distribution of the observed flux densities in 1999, 2000 and 2001. To avoid over-representation of certain values, data from the slow periods has been sampled according to the timescales calculated previously.

an increase in source size would always act to decrease the observed modulations. In the strong scattering regime, the modulation index $\propto 1 /($ source size) (Narayan 1992). We put a limit on the decrease in modulation index at $10 \%$, and thereby calculate that the source has expanded by less than $10 \%$ over two years.

\section{Properties of the scatterer and source size}

In Paper I we used a simple formula based on the observed timescale, the frequency at which the modulations were maximum $(\sim 5 \mathrm{GHz})$, and an assumed velocity through the scintillation pattern to deduce the location and strength of the scattering screen and the size of the source. (note a typographic error in Paper I: $\mathrm{C}_{N}^{2}$ calculated in that paper should read 0.017, not $0.17 \mathrm{~m}^{-20 / 3}$ ). We will return to this calculation again, now having ascertained the velocity through the scintillation pattern as a function of epoch. 
As the velocity of the plasma screen is of the order a few tens of $\mathrm{km} \mathrm{s}^{-1}$, and because of a lucky accident of the date of our first observations, the velocity used in Paper I was a reasonable figure, and therefore the measured velocity of the plasma screen does not itself change significantly the apparent size of the source. However, we also need to take in account the effects of the source size and the anisotropy in the scintillation pattern. These introduce a number of significant changes.

We reformulate the scattering results for a point source from Paper I, in terms of the desired quantities. These formulae are derived from Blandford \& Narayan (1985) and Romani et al. (1986), using the point of maximum refractive scattering ( $m=1)$, and assuming that the timescale is just the time taken to move through a scintle.

$$
\begin{aligned}
& \theta_{\mu \mathrm{as}}=64 \lambda_{\mathrm{cm}}^{\star} v_{\mathrm{kms}^{-1}}^{-1} t_{\mathrm{hrs}}^{-1} \\
& C_{-4}=1.2 \times 10^{8} \lambda_{\mathrm{cm}}^{\star} v_{\mathrm{kms}^{-1}}^{-11 / 3} t_{\mathrm{hrs}}^{-11 / 3} \\
& L_{\mathrm{kpc}}=1.9 \times 10^{-4} v_{\mathrm{kms}^{-1}}^{2} t_{\mathrm{hrs}}^{2} \lambda_{\mathrm{cm}}^{\star} .
\end{aligned}
$$

Here $C_{N}^{2}=10^{4} C_{-4}, \theta$ is the radius of the scattering disk, or the Fresnel disk at the critical frequency $\left(v^{\star}=c / \lambda^{\star}\right)$, and the distance to the equivalent screen is $L$, and we move with velocity $v$ through the scintillation pattern with timescale $t$. These equations can only be applied directly to observations at the critical frequency for a point source, or to a source with a non-scintillating base component and a point-like scintillating component.

As argued in Sect. 8.2 however, we consider the source to be resolved. The introduction of a source size means that the observables $\left(m, t, v^{\circ}\right)$ no longer reflect those of the refractive scintillations from a point source. We cannot therefore just drop the brightness temperature from that calculated in Paper I by a factor 4 to compensate for a source size increase of a factor 2 . In what follows we will turn the observed quantities into the equivalent point source values for use in the above equation, using the suffices ${ }^{\star}$ and ${ }^{\circ}$ to refer to quantities measured at the frequencies of maximum modulations for a point source, and for an extended source respectively. We refer to the frequency of maximum modulations for a point source, $v^{\star}$, as the "critical frequency".

\subsection{Effects of source size on $m$ and $t$ at maximum modulation}

A physical source would be expected to change its extent with observing frequency. This has an effect on the characteristics of the depth of modulation as a function of frequency if the source is resolved $\left(\theta_{\mathrm{S}}>\theta_{\text {scatt }}\right)$. An extended source does not exhibit maximum intensity variations at the same frequency as a point source, but at a frequency which is also dependent on its change of size with frequency. This is because the observed frequency of maximum modulations for a point source occurs when the scattering disk is the same size as the Fresnel zone, but for an extended source this occurs when the scattering disk is equal to the source size. This is illustrated in Fig. 15.

We are observing close to the frequency of maximum modulations. Simultaneous multifrequency observations from 10/2000 through 07/2001 confirm the effect reported in

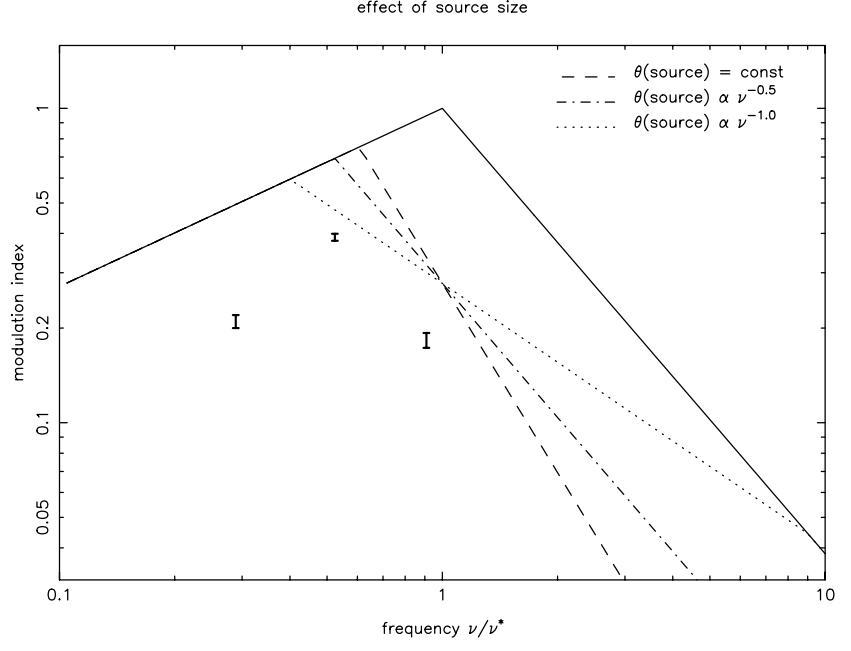

Fig. 15. The change of modulation index with frequency for source sizes larger than the scattering disk at the critical frequency, $v^{\star}$. The solid line represents a point source, the dotted lines extended sources, with frequency dependencies as indicated. All extended sources are 3 times the Fresnel zone at the critical frequency $\left(\theta_{\mathrm{F}}^{\star}\right)$. The points correspond to multifrequency observations, placed on the graph such that the $5 \mathrm{GHz}$ point is at $v^{\circ}$ for the case $\theta \propto v^{-0.5}$.

Paper I. The observations will be reported in a later paper, but the average modulation indices are plotted in Fig. 15, as it is critical to our analysis in this paper that the $5 \mathrm{GHz}$ observations are close to the modulation maximum. We can calculate the frequency of maximum modulations that would be observed for a point source, if we assume that the source is larger than the scattering disk in the observed frequency regime, and that there is no non-scintillating component.

We use the asymptotic theory (Narayan 1992), for both the strong and the weak regimes. Calculating the predicted modulation indices for a resolved source, we find that for source size decreasing with frequency less rapidly than $v^{-12 / 7}$, the observed frequency of maximum modulations is

$v^{\circ}=v^{\star}\left(m^{\circ}\right)^{30 / 17}$.

For illustration, the effect on the modulation index as a function of frequency for a number of source size frequency dependencies is illustrated in Fig. 15. The frequency dependence of the source size does not, however, affect the above relation.

Note that a non-scintillating component of the source would reduce the observed modulations without changing the observed modulation maximum from the critical frequency, but a resolved source results in a true critical frequency which is above the observed frequency of maximum modulation. This is because in this case, the maximum modulations do not occur at the transition from strong to weak scattering, but when the source size equals that of the scattering disk. Based on this simple calculation which assume the asymptotic theories are reasonable approximations to reality, and assuming there is no non-scintillating component, and using the observed maximum in the intensity modulations $\left(v^{\circ}\right)$ is around $5 \mathrm{GHz}$, we find that the critical frequency $\left(v^{\star}\right)$ is around $25 \mathrm{GHz}$.

We next consider the effect of source resolution on the observed timescale. Using the relation between the observed 
frequency of maximum modulation and the critical frequency derived above, we find that the timescale observed at the maximum modulations is related to the timescale for a point source at the critical frequency, $t^{\star}$, by

$t^{\circ}=\left(m^{\circ}\right)^{-66 / 17} t^{\star}$

Under the same assumptions as above, and using the observed $m^{\circ}=0.4$ we find $t^{\star}=t^{\circ} / 35$.

In order to relate the source size at the critical frequency to that at the frequency of observed modulation maximum, we must assume a dependency of source size on frequency. From multifrequency monitoring over the same period (forthcoming paper) we find that the spectral indices of the source around $5 \mathrm{GHz}$ are $\alpha_{2.3}^{4.9} \approx+1.0$ and $\alpha_{4.9}^{8.5} \approx+0.6$. We therefore expect that the source will display a frequency dependence between that of an optically thick source $(\alpha=+2.5, \theta$ constant) and a standard equipartition flat-spectrum source $(\alpha=0$, $\left.\theta \propto v^{-1}\right)$. We therefore consider a source which decreases in size as $v^{-0.5}$ (See also e.g. de Bruyn 1976).

In this case, and again for no non-scintillating component in the source, we find that the maximum modulation index, $m^{\circ}$, is related to the source size and the Fresnel scale at the critical frequency as

$m^{\circ}=\left(\theta_{\mathrm{F}}^{\star} / \theta_{\mathrm{S}}^{\star}\right)^{1 / 3}$

Using the observed $m^{\circ}=0.4$, we find $\theta_{\mathrm{S}}^{\star}=15 \theta_{\mathrm{F}}^{\star}$. Thus the source could be up to 15 times the size of the Fresnel scale, at the critical frequency, i.e. at $25 \mathrm{GHz}$.

\subsection{Effects of anisotropy}

Finally we must account for the anisotropy. Without consideration of the anisotropy we cannot turn the observed values into source and screen parameters, because of the order of magnitude change in observed timescale during a year. The interpretation of the anisotropy is the most difficult, and potentially the biggest source of error as the theory used is an isotropic scattering theory. In what follows we simply take geometric means of relevant quantities, and assume these would be the values appropriate to an isotropic theory. We consider two cases: that the anisotropy is due entirely to the medium, or entirely due to the source. It is possible that both the effects play a role: in this case the position angle of the anisotropy fitted to the data represents a position angle of the convolution of the two effects.

If the anisotropy is due to the scattering medium, it is not clear how we should take this into account, as a full treatment of anisotropic scattering should be used, not the isotropic case on which we base our arguments. It is complicated by the fact that, as argued above, the source is likely to have a size comparable to the scattering scales. To make a step towards accounting for the anisotropy, we consider the situation when the Earth cuts through the anisotropy such that the scale is the geometric mean of the two scales (day 220, for best fit $\epsilon=14$ ), when our velocity through the scintillation pattern is calculated to be $54 \mathrm{~km} \mathrm{~s}^{-1}$.

\subsection{Derived source and screen parameters}

First we consider that the medium is isotropic and the source is a resolved ellipsoid with no non-scintillating base component. Because of the conversion estimates we are making to treat the isotropic theory in a way to account for anisotropy, the results will also be those for the case in which the observed anisotropy is due to the medium, but the source itself is a partially resolved isotropic source.

From the above considerations of the effect of source size we find $t^{\star}=t^{\circ} / 35=0.057 \mathrm{hrs}$. We note that here we associate twice the timescales measured and presented in Table 3 with the time required in the formula, and the angular scales as corresponding to the diameter of the source. From the preceding discussion, we associate $t$ with $t^{\star}$ and $\theta$ with $\theta_{\mathrm{F}}^{\star}$ in the formulae given at the beginning of Sect. 9. Using $v=54 \mathrm{~km} \mathrm{~s}^{-1}$, $m^{\circ}=0.4, v^{\circ}=5 \mathrm{GHz}$ in these formulae, we find $\theta_{\mathrm{F}}^{\star}=25 \mu$ as. At $5 \mathrm{GHz}$ the source is then $\theta_{\mathrm{F}}^{\star} \times 15 \times\left(v^{\star} / v\right)^{0.5}=860 \mu$ as. The source has a mean flux density of $200 \mathrm{mJy}$, resulting in a brightness temperature $T_{\mathrm{B}}=2 S \lambda^{2} / \pi k \theta_{\mathrm{S}}^{2}=1.3 \times 10^{10} \mathrm{~K}$. In this case the screen is extremely strong $\left(C_{N}^{2} \approx 165 \mathrm{~m}^{-20 / 3}\right)$, and nearby $(\sim 1 \mathrm{pc})$, which results in the larger angle subtended by the scattering disks. This in turn considerably lowers the brightness temperature from that calculated in Paper I.

We now consider the case of the maximum possible flux density in a non-scintillating component. Although the modulation index is 0.4 , we know that $>40 \%$ of the source is scintillating, by the regular minima at $\sim 60 \mathrm{mJy}$. We therefore consider, in a similar manner to Paper I a non-scintillating baselevel determined by this value. From Fig. 14a we take $140 \mathrm{mJy}$ in the scintillating component. However our approach here differs from Paper I, not just due to our revised velocity, and calculated anisotropy, but because we take into account that this scintillating component must also be somewhat resolved. The reason for this is that the modulation index is not 0.7 , as would be expected from scintillation of a $140 \mathrm{mJy}$ point source at the critical frequency, on a non-scintillating component of $60 \mathrm{mJy}$. The observed modulation index of the smaller component, i.e. if we remove the base component, is $m^{\circ \prime}=m^{\circ} / x$, where $x$ is the fraction of the total flux density in the scintillating component. We use $m^{\circ \prime}$ in place of $m^{\circ}$ in the formulae given in the preceding sections. Again using $v=54 \mathrm{~km} \mathrm{~s}^{-1}, t^{\circ}=2 \mathrm{hrs}$, and $v^{\circ}=5 \mathrm{GHz}$, we obtain at $5 \mathrm{GHz}$ a source size of $100 \mu$ as and brightness temperature of $7 \times 10^{11} \mathrm{~K}$. The scattering screen is $12 \mathrm{pc}$ away with $C_{N}^{2}=0.5 \mathrm{~m}^{-20 / 3}$.

We have presented the boundary scenarios: the reality may lie somewhere in between. Figure 16 shows the two extreme cases discussed above ( $x=1$ and 0.7$)$, as well as the intermediary cases. We note that the size quoted is the effective diameter, so for an axial ratio of $6: 1$, the source would be $\sqrt{6} \approx 2.5$ times this in the direction of the elongation, and $1 / \sqrt{6} \approx 0.4$ times this orthogonally to it.

Our observed $v^{\circ}$ at around $5 \mathrm{GHz}$ falls near to the critical frequency predicted from contributions to scattering from the entire Galaxy (Walker 1998), but this must be coincidental because, aside from the reasons given in this section, the peculiar velocity rules out a contributions from a very extended material, as does the slope of the structure function. 


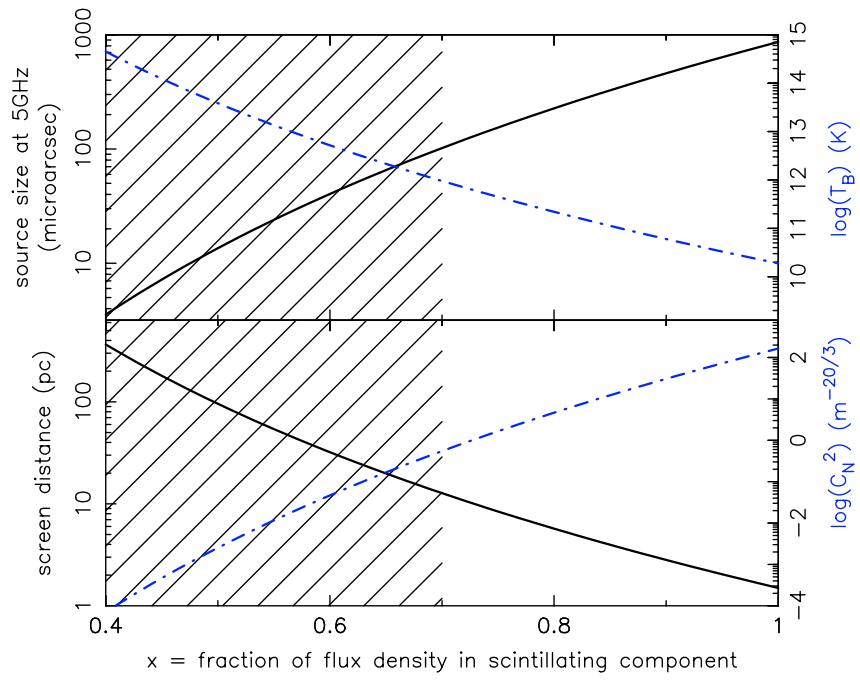

Fig. 16. The derived range of source and screen properties (upper and lower panel respectively), taking into account the effects of source resolution on the observed timescale and modulation index at the frequency of maximum variation. The solid lines refer to the scale on the left, the dotted lines to that on the right. We require that the combined effects of the non-scintillating component and the size of the scintillating component result in the observed maximum modulation index, and assume $\theta \propto v^{-0.5}$. The values at $x=0.4$ correspond to a pointlike $\left(\theta_{\mathrm{S}}<\theta_{\text {scatt }}\right)$ component on a non-scintillating base. However, the left-hand side (shaded) is not compatible with other considerations of the data: the PDFs and the original lightcurves show this is not compatible with the deep minima: $\geq 70 \%$ of the flux density must be in a scintillating component.

We calculate a high critical frequency of between 13 and $25 \mathrm{GHz}(0.7<x<1)$. In this particular direction we see that any source brighter than a hundred mJy at $5 \mathrm{GHz}$ would have to have a $T_{\mathrm{B}}$ far in excess of the Compton limit if it were to demonstrate an observed modulation maximum around $25 \mathrm{GHz}$. The coincidence is probably also partly a reflection of the more frequent observing at $\mathrm{GHz}$ frequencies, where this source is most variable.

We consider also the possibility of the source as modelled by two well-separated sources, each smaller than the scattering disk but separated by several times this. Such a situation is similar to some structures observed in VLBI images of corejets and compact radio galaxies. Motion parallel to the axis of separation of the components would produce a conspicuous "dip" in the rising portion of the structure function (Spangler et al. 1993). The nature of the observed structure functions for $\mathrm{J} 1819+3845$ indicates this is unlikely, as the fit indicate most observations as well as most scintles are observed during motion suitable to see the development of these two timescales. There is no such evidence for this: the "overshoot" of the structure function cannot be explained in these terms, as the structure function reaches saturation values before turning over. We therefore rule out a model of discrete and well-separated source components.

Finally we note that, as well as not using anisotropic scattering theory, all these calculations are based on asymptotic theory which is not strictly applicable in this regime. Further work testing the theory with simulations near the critical frequency is required, as well as the effects on the observables of partially and slightly resolved sources $\left(\theta_{\mathrm{S}} \approx \theta_{\text {scatt }}\right)$. Only more careful theoretical work will enable the scattering material and source size to be properly constrained. At present, even for a source with very well defined characteristics (a strong limit on "non-scintillating" components, and a known velocity of the scattering screen) the constraints on source size are not tight.

It can be seen that the brightness temperature can be below the Compton limit, but only if the scatterer is very strong and only a few parsecs from Earth. Regardless of the scattering theory, we note that if the source has a $T_{\mathrm{B}}<5 \times 10^{11} \mathrm{~K}$, the source must be $>140 \mu$ as in diameter. The detection of anisotropy, whether in scatterer or source, is a robust result which does not depend on theoretical details. The anisotropy required by the annual modulation fits has an axial ratio of $>6: 1$, and if this is due to source structure, the longest axis must be $>0.34$ mas at $5 \mathrm{GHz}$. This is resolvable by space VLBI or ground-based VLBI at higher frequencies. Global VLBI observations at $22 \mathrm{GHz}$ (Dennett-Thorpe, Gurvits et al., in prep.) show $\mathrm{J} 1819+3845$ to be $<0.4$ mas at this frequency. If we assume that the source at $22 \mathrm{GHz}$ closely related to that at $5 \mathrm{GHz}$, in particular the (not unreasonable) assumption that there are no milliarcsecond components which are present at $5 \mathrm{GHz}$ and not at $22 \mathrm{GHz}$, we determine an upper limit on the source size at $5 \mathrm{GHz}$. Using the relation source size $\propto v^{-0.5}$, we find the major axis of the source at $5 \mathrm{GHz}$ is $<0.8$ mas, which is an equivalent diameter for axial ratio $6: 1$ of $<0.32$ mas. From this we favour a source which exceeds $5 \times 10^{11} \mathrm{~K}$ at $5 \mathrm{GHz}$. Combined with the preceeding discussion this would put the screen at a distance between 4 and $12 \mathrm{pc}$.

\section{Concluding remarks}

We have presented observations over a two year period for the quasar J1819+3845. Notable results of this study were:

(i) The source continues to vary throughout the two years. Although the timescale of these variation changes by a factor of ten or more, the strength of the modulation does not appear to change.

(ii) The change in timescale has a periodicity of a year, and can be explained by a velocity of the plasma screen and an additional component, which is due to source structure or anisotropic turbulence in the scattering plasma. These observations do not allow us to uniquely determine all four parameters, although $v_{\mathrm{dec}}$ of the scattering plasma and the position angle of the anisotropy are well constrained. Combining these observations with the results of our previous experiment (Dennett-Thorpe \& de Bruyn 2001, Paper II) where we measured the velocity of the plasma screen directly, we constrain the axial ratio of the source, or the turbulent cells to be $>6: 1$ and oriented at $\mathrm{PA}=83^{\circ} \pm 4^{\circ}$.

(iii) The source is resolved, as can be seen by the smoothness of the modulations. From the depth of the modulations, we deduce that the source at $5 \mathrm{GHz}$ is at most a few times the scattering disk at the scattering screen. 
(iv) Based on the interpretation of the structure function, we determine that the plasma responsible for scattering is a thin screen.

(v) Any source expansion has been $<10 \%$ over the two years of monitoring. No significant change in structure has been detected.

(vi) The source has been getting brighter at $5 \mathrm{GHz}$. The increase in flux density has been $\sim 25 \% /$ year. Coupled with the lack of source expansion, this $50 \%$ increase in flux density, must be largely due to an increase in the brightness temperature.

Interstellar scintillation has already been proved to be the cause of the variations in J1819+3845 (Paper II), but this paper shows that monitoring observations over a year can also be a proof of scintillation. Since this project started this method has been applied to B 0917+624 (Rickett et al. 2001; Jauncey \& Macquart 2001), although using a very few points. Monitoring campaigns are more appropriate for the majority of IDV sources, as their timescales of modulation are not short enough to be able to conduct a two-telescope delay experiment. Furthermore, we demonstrate that the transverse velocity of the scattering plasma can be calculated using this method: to our knowledge this is the first such measurement.

Using the determined plasma velocity we obtain an equivalent angular source size of between 100 and 900 microarcsec, and corresponding brightness temperatures in the range $10^{10}$ and $10^{12} \mathrm{~K}$. In this calculation we consider the effects of the spatial distribution of the scintillating flux - how much of the source could be smaller than the scattering disk. This consideration is the main reason for a decrease in $T_{\mathrm{B}}$ from $10^{14} \mathrm{~K}$ calculated in Paper I, although we still favour a source $T_{\mathrm{B}}>$ $5 \times 10^{11} \mathrm{~K}$. The scattering material is very unusual: it is very strong and between 1 and 12 parsecs away.

Modelling of the source extent in relation to the the scattering disk is a critical factor in determining both the intrinsic source size and the parameters of the scattering medium, and we are undertaking work on this. Although we have taken account of source resolution, we have not used a full and proper approach to deal with the anisotropy, and this also needs to be addressed.

This work emphasises that consideration of the effects of source extension could have a very big impact on the parameters derived. In the meantime we must be wary of using models of scintillating and non-scintillating components to describe scintillating extragalactic radio sources, as the source sizes and brightness temperatures obtained in such a way are very poor estimates.

The detection of the anisotropy is a robust result which does not depend on the details of the theory, but whether the anisotropy is due to the source itself, or to the scattering material remains an open question.

Acknowledgements. This research was supported by the European Commission, TMR Programme, Research Network Contract ERBFMRXCT96-0034 "CERES" and NOVA. The William Herschel Telescope is operated on the island of La Palma by the Isaac Newton Group in the Spanish Observatorio del Roque de los Muchachos of the Instituto de Astrofisica de Canarias. The WSRT is operated by the Netherlands Foundation for Research in Astronomy (NFRA/ASTRON) with financial support by The Netherlands Organization for Scientific Research (NWO).

We wish to thank Frank Briggs for providing us with the solution to the 3D trigonometry; Rene Vermeulen for generous scheduling at the WSRT especially in the early stages of the project; and Barney Rickett and J.-P. Marquart for helpful discussions. JDT also thanks the Astronomical Institute Anton Pannekoek, University of Amsterdam for their hospitality, and The Netherlands Research School for Astronomy (NOVA) for support during the completion of this work.

\section{References}

Aller, H. D., Aller, M. F., Latimer, G. E., \& Hodge, P. E. 1985, ApJS, 59,513

Armstrong, J. W., Coles, W. A., Rickett, B. J., \& Kojima, M. 1990, ApJ, 358, 685

Baker, J. C. 1997, MNRAS, 286, 23

Blandford, R., \& Narayan, R. 1985, MNRAS, 213, 591

Blandford, R., Narayan, R., \& Romani, R. W. 1986, ApJ, 301, L53

Bondi, M., Padrielli, L., Fanti, R., et al. 1996, A\&A, 308, 415

Brotherton, M. S., Tran, H. D., Becker, R. H., et al. 2001, ApJ, 546, 775

Chandran, B., \& Backer, D. 2002, ApJ, 576, 176

Cohen, M. H., Gundermann, E. J., \& Harris, D. E. 1967, ApJ, 150, 767

de Bruyn, A. G. 1976, A\&A, 52, 439

de Bruyn, A. G., \& Dennett-Thorpe, J. 2001, Ap\&SS, 278, 139

Dennett-Thorpe, J., \& de Bruyn, A. G. 2000, ApJ, 529, L65

Dennett-Thorpe, J., \& de Bruyn, A. G. 2001, Nature, 415, 57

Deshpande, A. A., \& Nityananda, R. 1990, A\&A, 231, 199

Frail, D. A., Diamond, P. J., Cordes, J. M., \& van Langevelde, H. J. 1994, ApJ, 427, L43

Gupta, Y., Rickett, B. J., \& Lyne, A. G. 1994, MNRAS, 269, 1035

Heeschen, D. S., Krichbaum, T., Schalinski, C. J., \& Witzel, A. 1987, AJ, 94, 1493

Hewish, A., Scott, P., \& Wills, D. 1964, Nature, 203, 1214

Hunstead, R. W. 1972, Astrophys. Lett., 12, 193

Jauncey, D. L., \& Macquart, J.-P. 2001, A\&A, 370, L9

Kedziora-Chudczer, L., Jauncey, D. L., Wieringa, M. H., et al. 1997, ApJ, 490, L9

Kraus, A., Witzel, A., Krichbaum, T. P., et al. 1999, A\&A, 352, L107

Lithwick, Y., \& Goldreich, P. 2001, ApJ, 562, 279

Lo, K. Y., Backer, D. C., Kellermann, K. I., et al. 1993, Nature, 362, 38

Miller, P., Rawlings, S., Saunders, R., \& Eales, S. 1992, MNRAS, 254, 93

Narayan, R. 1992, Phil. Trans. R. Soc. Lond. A, 341, 151

Narayan, R., Anantharamaiah, K. R., \& Cornwell, T. J. 1989, MNRAS, 241, 403

Noordam, J. 1994, The NEWSTAR cookbook, Internal NFRA report

Peng, B., Kraus, A., Krichbaum, T. P., et al. 2000, A\&A, 353, 937

Pohl, M., Reich, W., Schlickeiser, R., Reich, P., \& Ungerechts, H. 1996, A\&AS, 120, C529

Qian, S. J., Quirrenbach, A., Witzel, A., et al. 1991, A\&A, 241, 15

Quirrenbach, A., Kraus, A., Witzel, A., et al. 2000, A\&AS, 141, 221

Quirrenbach, A., Witzel, A., Qian, S. J., et al. 1989, A\&A, 226, L1

Readhead, A. C. S., \& Hewish, A. 1976, MNRAS, 176, 571

Rickett, B. J. 1990, ARA\&A, 28, 561 
Rickett, B. J. 2001, Ap\&SS, 278, 129

Rickett, B. J., Coles, W. A., \& Bourgois, G. 1984, A\&A, 134, 390

Rickett, B. J., Coles, W. A., \& Markkanen, J. 2000, ApJ, 533, 304

Rickett, B. J., Quirrenbach, A., Wegner, R., Krichbaum, T. P., \& Witzel, A. 1995, A\&A, 293, 479

Rickett, B. J., Witzel, A., Kraus, A., Krichbaum, T. P., \& Qian, S. J. 2001, ApJ, 550, L11

Romani, R. W., Narayan, R., \& Blandford, R. 1986, MNRAS, 220, 19

Shapirovskaya, N. J. 1991, Pisma Astronomicheskii Zh., 17, 897

Simonetti, J. H. 1991, A\&A, 250, L1

Simonetti, J. H., Cordes, J. M., \& Heeschen, D. S. 1985, ApJ, 296, 46 Spangler, S., Fanti, R., Gregorini, L., \& Padrielli, L. 1989, A\&A, 209, 315
Spangler, S. R., Eastman, W. A., Gregorini, L., Mantovani, F., \& Padrielli, L. 1993, A\&A, 267, 213

Stevens, J. A., Litchfield, S. J., Robson, E. I., et al. 1995, MNRAS, 275,1146

Trotter, A. S., Moran, J. M., \& Rodriguez, L. F. 1998, ApJ, 493, 666

Wagner, S. J., \& Witzel, A. 1995, ARA\&A, 33, 163

Wagner, S. J., Witzel, A., Heidt, J., et al. 1996, AJ, 111, 2187

Walker, M. A. 1998, MNRAS, 294, 307

Wilkinson, P. N., Narayan, R., \& Spencer, R. E. 1994, MNRAS, 269, 67

Willott, C. J., Rawlings, S., Blundell, K. M., \& Lacy, M. 1999, MNRAS, 309, 1017 